Draft Version November 5, 2020

Typeset using LATEX twocolumn style in AASTeX63

\title{
Identification of Methyl Isocyanate and Other Complex Organic Molecules in a Hot Molecular Core, G31.41+0.31
}

\author{
Prasanta Gorai, ${ }^{1,2}$ Ankan Das,${ }^{2}$ Takashi Shimonishi, ${ }^{3,4}$ Dipen Sahu, ${ }^{5}$ Suman Kumar Mondal, ${ }^{2}$ Bratati Bhat, ${ }^{2}$ And \\ SANDip K. Chakrabarti ${ }^{2}$ \\ ${ }^{1}$ Department of Space, Earth $\&$ Environment, Chalmers University of Technology, SE-412 96 Gothenburg, Sweden \\ 2 Indian Centre for Space Physics, 43 Chalantika, Garia Station Road, Kolkata 700084, India \\ ${ }^{3}$ Center for Transdisciplinary Research, Niigata University, Ikarashi-ninocho 8050, Nishi-ku, Niigata, 950-2181, Japan \\ ${ }^{4}$ Environmental Science Program, Department of Science, Faculty of Science, Niigata University, Ikarashi-ninocho 8050, Nishi-ku, \\ Niigata, 950-2181, Japan \\ ${ }^{5}$ Academia Sinica Institute of Astronomy and Astrophysics, $11 \mathrm{~F}$ of AS/NTU Astronomy-Mathematics Building, No.1, Section 4, \\ Roosevelt Rd, Taipei 10617, Taiwan, R.O.C.
}

\begin{abstract}
G31.41+0.31 is a well known chemically rich hot molecular core (HMC). Using Band 3 observations of Atacama Large Millimeter Array (ALMA), we have analyzed the chemical and physical properties of the source. We have identified methyl isocyanate $\left(\mathrm{CH}_{3} \mathrm{NCO}\right)$, a precursor of prebiotic molecules, towards the source. In addition to this, we have reported complex organic molecules (COMs) like methanol $\left(\mathrm{CH}_{3} \mathrm{OH}\right)$, methanethiol $\left(\mathrm{CH}_{3} \mathrm{SH}\right)$, and methyl formate $\left(\mathrm{CH}_{3} \mathrm{OCHO}\right)$. Additionally, we have used transitions from molecules like $\mathrm{HCN}, \mathrm{HCO}^{+}, \mathrm{SiO}$ to trace the presence of infall and outflow signatures around the star-forming region. For the COMs, we have estimated the column densities and kinetic temperatures, assuming molecular excitation under local thermodynamic equilibrium (LTE) conditions. From the estimated kinetic temperatures of certain COMs, we found that multiple temperature components may be present in the HMC environment. Comparing the obtained molecular column densities between the existing observational results toward other HMCs, it seems that the COMs are favourably produced in the hot-core environment ( $\sim 100 \mathrm{~K}$ or higher). Though the spectral emissions towards G31.41+0.31 are not fully resolved, we find that $\mathrm{CH}_{3} \mathrm{NCO}$ and other COMs are possibly formed on grain/ice phase and populate the gas environment similar to other hot cores like Sgr B2, Orion KL, and G10.47+0.03, etc.
\end{abstract}

Keywords: astrochemistry-line: identification-ISM: individual(G31.41+0.31)-ISM: molecules

\section{INTRODUCTION}

Chemistry in high-mass star-forming regions (HMSFRs) is very rich, and it has a significant impact on the evolution of the Interstellar Medium (ISM) (e.g., van Dishoeck \& Black et al. 1998; Tan et al. 2014). Over the years, numerous complex organic molecules (COMs) were identified in hot molecular cores (HMCs) (e.g., Herbst \& van Dishoeck 2009). Hot molecular cores are compact, hot, dense, and associated with luminous infra-red (IR) or with ultra-compact (UC) HII regions (Cesaroni et al. 2010; Bonfand et al. 2017). To date, a number of HMCs were discovered (Kurtz et al. 2000),

ankan.das@gmail.com,

prasanta.astro@gmail.com while a limited number of circumstellar disks were found around B type stars such as the disks in Cepheus A HW2 (Patel et al. 2005), HH80-81 (Girart et al. 2018) and the disk in the late-O-type star, IRAS 13481-6124 (Kraus et al. 2010). Their dust emission has identified the majority of these disk structures. However, they are also associated with molecular emission. Complex organic molecules, $\mathrm{CH}_{3} \mathrm{CN}$, was observed in Cepheus A HW2 and $\mathrm{P}-\mathrm{V}$ diagram of $\mathrm{CH}_{3} \mathrm{CN}$ transitions used to understand the velocity gradient that explains gravitationally bound rotational motion.

Many complex physical processes, such as accretion, infall, and outflows are present in the earliest phase of HMSFRs during their various evolutionary stages. Therefore the chemistry of these regions is used as a diagnostic tool to examine the nature of them. Complex organic molecules are omnipresent in the HMCs and 
show rich chemistry. Chemistry of these regions plays an essential role, which helps trace the physical parameters associated with particular regions, such as density, temperature, and ionization rate. Since the abundances of chemical species are time dependent and collapsing time of a HMSFR is short, a comparison of observed abundance with modeling can be used to trace the chemical diversity of those region. For HMCs, both grain-surface and gas-phase processes are efficient. Various desorption mechanisms, such as thermal (active in hot core temperature) and non-thermal desorption (dominant at low temperature) processes help release the grain surface species to the gas phase.

G31.41+0.31 (hereafter G31) is an HMC that is thought to be located at a $7.9 \mathrm{kpc}$ distance from the Earth (Churchwell et al. 1990). This source has a luminosity of about $\sim 3 \times 10^{5} L_{\odot}$. It is supposed to be heated by $\mathrm{O}$ and $\mathrm{B}$ type stars (Cesaroni et al. 2010; Beltrán et al. 2009). Previous studies (Cesaroni et al. 2011, 2017) found some hints of the existence of disk in G31.41+0.31. Osorio et al. (2009) performed detailed modeling of the SED of dust to obtain the physical parameters of the star and the core. Using these parameters, they estimated the ammonia emission and compared it with the VLA observation having a sub-arcsec resolution of G31. They obtained the mass of the central star $\sim 20-25 \mathrm{M}_{\odot}$ and significantly higher mass (1400$1800 \mathrm{M}_{\odot}$ ) of the envelope by considering envelope size, $\mathrm{R}_{\text {env }}=30000$ AU. Recently, Beltrán et al. (2018) observed G31 with ALMA at $1.4 \mathrm{~mm}$ resolved G31 into two cores, namely main and NE. They observed the physical properties such as accelerating infall, rotational spin up, red-shifted absorption, and possible outflow directions associated with this source. The UC HII region is situated at the North-East side of G31 and separated by $\sim 5^{\prime \prime}$ from the main core (Cesaroni et al. 2010). The main core's estimated mass is $120 \mathrm{M}_{\odot}$, which is based on old distance $7.9 \mathrm{kpc}$ (Beltrán et al. 2018). However, new parallax measurement suggests that G31 is located at a distance of $3.7 \mathrm{kpc}$. Therefore the luminosity of the source would be $\sim 4 \times 10^{4} L_{\odot}$ and estimated mass of the main core $\left(M_{c}\right)$ of $\mathrm{G} 31$ is $\sim 26 \mathrm{M}_{\odot}$ (Ried et al. 2019; Estalella et al. 2019; Beltrán et al. 2019). However, this estimation should be considered the lower limit because high angular resolution might have filtered spatially extended emission (Beltrán et al. 2019).

A wide variety of species (around 70) were already identified in G31. The simplest form of sugar, glycolaldehyde $\left(\mathrm{HOCH}_{2} \mathrm{CHO}\right)$, was observed in $\mathrm{G} 31$ with IRAM PdBI at 1.4, 2.1, and $2.9 \mathrm{~mm}$ observations (Beltrán et al. 2009). This is the earliest evidence of the presence of sugar outside our Galactic center. A large number of COMs such as methanol $\left(\mathrm{CH}_{3} \mathrm{OH}\right)$, methyl cyanide $\left(\mathrm{CH}_{3} \mathrm{CN}\right)$, ethanol $\left(\mathrm{C}_{2} \mathrm{H}_{5} \mathrm{OH}\right)$, ethyl cyanide $\left(\mathrm{C}_{2} \mathrm{H}_{5} \mathrm{CN}\right)$, methyl formate $\left(\mathrm{CH}_{3} \mathrm{OCHO}\right)$, dimethyl ether $\left(\mathrm{CH}_{3} \mathrm{OCH}_{3}\right)$, ethylene glycol $\left(\mathrm{CH}_{2} \mathrm{OH}\right)_{2}$, and acetone $\left(\mathrm{CH}_{3} \mathrm{COCH}_{3}\right)$ had already been identified in G31 by using both single dish (IRAM 30m, GBT) and Submillimeter Array (SMA) observations (Rivilla et al. 2017; Isokoski, Bottinelli \& van Dishoeck. 2013, and references therein).

The presence of a large number of COMs, including branched chain molecule might be an indication of the existence of prebiotic molecules and essential building blocks of life in space (Belloche et al. 2014; Chakrabarti et al. 2015; Majumdar et al. 2015; Sil et al. 2018; Sahu et al. 2020). Goesmann et al. (2015) detected various COMs (e.g., methyl isocyanate, formamide, glycolaldehyde, ethylamine etc.) in a Jupiter family comet, 67P/Churyumov-Gerasimenko (67P/C$\mathrm{G})$. Among them, methyl isocyanate $\left(\mathrm{CH}_{3} \mathrm{NCO}\right)$ was found to be relatively abundant as compared to other observed COMs. However, Altwegg et al. (2017) reported the resulting $\mathrm{CH}_{3} \mathrm{NCO}$ abundance as an upper limit. The presence of $\mathrm{CH}_{3} \mathrm{NCO}$ in space is supposed to be important because of its peptide-like bond. Detection of $\mathrm{CH}_{3} \mathrm{NCO}$ was first claimed by Halfen et al. (2015) in a massive hot molecular core, Sgr B2. It was also detected in Orion KL (Cernicharo et al. 2016). It has recently been observed in G10.47+0.03 (Gorai et al. 2020). Here, in G31, we have identified some transitions of this species.

Methanethiol $\left(\mathrm{CH}_{3} \mathrm{SH}\right)$ is a sulfur analog of methanol, where the $\mathrm{S}$ atom is replaced by an $\mathrm{O}$ atom. To date, this is the only complex sulfur-bearing species (having 6 atoms) which has been firmly detected in the ISM. Tentative identification of higher-order thiols, ethanethiol $\left(\mathrm{C}_{2} \mathrm{H}_{5} \mathrm{SH}\right)$ has been reported in Sgr B2N (Müller et al. 2016). Identification of $\mathrm{CH}_{3} \mathrm{SH}$ was also reported toward the Galactic center (Linke et al. 1979; Müller et al. 2016), Orion KL (Kolesniková et al. 2014) and chemically rich massive hot core, G327.3-6 (Gibb et al. 2000) and 67P/C-G comet (Calmonte et al. 2016).

In this work, we present the interferometric (ALMA archival data) observation towards G31. We report first detection of $\mathrm{CH}_{3} \mathrm{NCO}$ and $\mathrm{CH}_{3} \mathrm{SH}$ in $\mathrm{G} 31$ and discuss the kinematics associated with this HMC. We also discuss the chemistry of other complex species in this source. The remainder of this paper is summarized as follows. In Section 2, we describe observational details and data reduction processes. All results are presented and discussed in Section 3 and Section 4 respectively. Finally, we draw our conclusion in Section 5. 


\section{ALMA ARCHIVAL DATA}

In this paper, we used Atacama Large Millimeter/submillimeter Array (ALMA) cycle 3 archival data (\#2015.1.01193.S) to study COMs in G31. The observation was performed during June, 2016 using forty $12 \mathrm{~m}$ antennas. The shortest and the longest baseline was 15 $\mathrm{m}$, and $783.0 \mathrm{~m}$, respectively, and synthesized beam size was $\sim 1.1^{\prime \prime}$. Four spectral windows $(86.559-87.028 \mathrm{GHz}$, 88.479-88.947 GHz, 98.977-99.211 GHz, and 101.076$101.545 \mathrm{GHz}$ ) of width $0.47 \mathrm{GHz}$ was set up to observe the target source, G31; phase center was $\alpha(\mathrm{J} 2000)$ $=18^{h} 47^{m} 34.309^{s}, \delta=-1^{o} 12^{\prime} 46.00^{\prime \prime}$. The flux calibrator was Titan, the phase calibrator was J1824+0119, and the bandpass calibrator was J1751+0939. The data cube has a spectral resolution of $244 \mathrm{kHz}(\sim 0.84 \mathrm{~km}$ $\mathrm{s}^{-1}$ ) with a synthesized beam of $\sim 1.19^{\prime \prime} \times 0.98^{\prime \prime}$ having position angle $(\mathrm{PA}) \sim 76^{\circ}$. The systematic velocity of the source is known to be $\sim 97 \mathrm{~km} \mathrm{~s}^{-1}$ (Cesaroni et al. 2010; Rivilla et al. 2017). Image analysis was done using CASA 4.7.2 software (McMullin et al. 2007). Implementing the first-order baseline fit and by using 'uvcontsub' command available in the CASA program, we separated each spectral window into two data: continuum and line emission. The identification of lines of all the observed species presented in this paper is carried out using CASSIS (This software was developed by IRAP-UPS/CNRS, http://cassis.irap.omp.eu) together with the spectroscopic database 'Cologne Database for Molecular Spectroscopy' (CDMS, Müller et al. 2001, 2005) ${ }^{1}$ and Jet Propulsion Laboratory (JPL, Pickett et al. 1998) ${ }^{2}$ database.

\section{RESULTS}

\subsection{Continuum emission and $\mathrm{H}_{2}$ column density estimation}

The continuum image of G31 at $94.3 \mathrm{GHz}$ is shown in Figure 1. The synthesized beam size of the continuum is $1.19^{\prime \prime} \times 0.98^{\prime \prime}$ with $\mathrm{PA} \sim 76^{\circ}$. Using two-dimensional Gaussian fitting over the dust continuum emission, we obtained the deconvolved size of G31 0.90" $\times 0.68^{\prime \prime}$.

Assuming the distance to the source $7.9 \mathrm{kpc}$, we obtained a source size $\sim 7110 \times 5372 \mathrm{AU}$. This source size changes to $3330 \times 2516 \mathrm{AU}$, while $\sim 3.7 \mathrm{kpc}$ distance is used.

Beltrán et al. (2018) observed G31 with an angular resolution of $0.22^{\prime \prime}$ and found the continuum brightness temperature $\sim 132 \mathrm{~K}$, which is comparable to the dust temperature $(\sim 150 \mathrm{~K})$. This suggests that the dust con-

\footnotetext{
1 (https://www.astro.uni-koeln.de/cdms)

2 (http://spec.jpl.nasa.gov/)
}

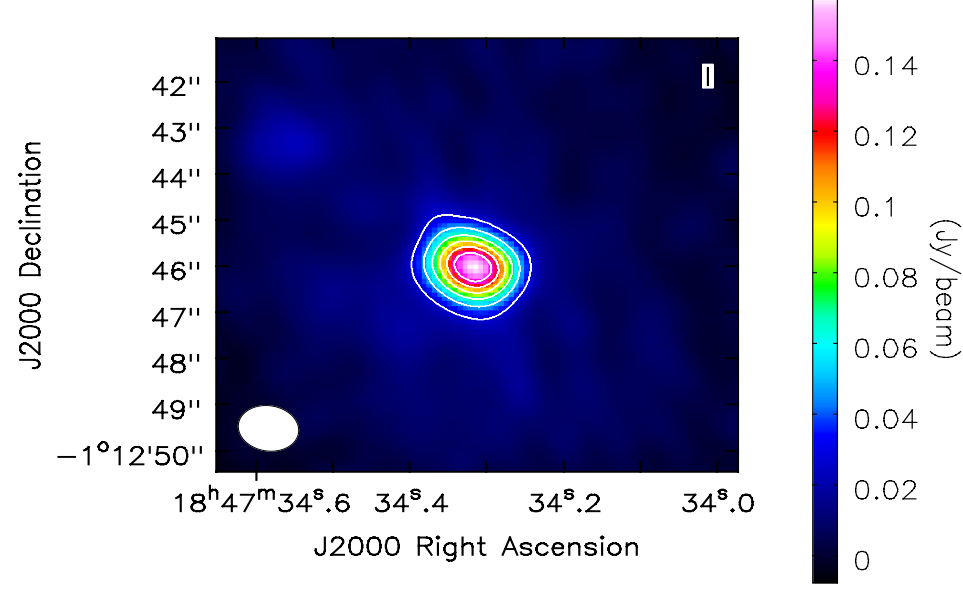

Figure 1. Continuum map of G31 obtained with ALMA at $94 \mathrm{GHz}$. Contour levels start at $4 \sigma(1 \sigma=7 \mathrm{mJy} / \mathrm{beam})$ and steps are in $3 \sigma$. Ellipse at the below left corner of the image shows the synthesized beam $\left(1.19^{\prime \prime} \times 0.98^{\prime \prime}\right)$ with $\mathrm{PA}=76.83^{\circ}$.

tinuum emission observed at $217 \mathrm{GHz}$ is optically thick. Rivilla et al. (2017) also observed that dust opacity is high $(\tau=2.6)$ at $220 \mathrm{GHz}$.

We have observed peak intensity of the continuum is $\sim 158 \mathrm{mJy} /$ beam which corresponds to a brightness temperature $18 \mathrm{~K}$ (using Rayleigh-Jeans approximation, $1 \mathrm{Jy} /$ beam $\equiv 118 \mathrm{~K}$ ). This estimation is much smaller than the assumed dust temperature of $150 \mathrm{~K}$, this indicates a small optical depth $\left(\tau_{\nu}\right) \sim 0.12$ (using $T_{m b}=T_{d}\left(1-\exp \left(-\tau_{\nu}\right)\right)$.

It should be noted that in our observation, the source was not resolved or at best marginally resolved; therefore, the estimated brightness temperature could be underestimated.. After adding the beam dilution correction for the unresolved source, the brightness temperature is estimated to be $\sim 36 \mathrm{~K}$. Considering this value of brightness temperature; dust emission remains optically thin $\left(\tau_{\nu} \sim 0.24\right)$. Due to the collapsing envelope, we can expect higher density and temperature toward the inner region than the outer envelope of this source. Since dust density is higher towards the inner region, opacity would be higher, as observed by Beltrán et al. (2018). It may reduce the true line intensity of the observed transitions. However, due to the lower angular resolution, our present data would not provide further details about the dust opacity of the whole region of G31. 
Assuming the dust continuum to be optically thin, flux density can be written as,

$$
S_{\nu}^{\text {beam }}=\Omega_{\text {beam }} \tau_{\nu} B_{\nu}\left(T_{d}\right),
$$

where $\Omega_{\text {beam }}=\frac{\pi}{4 \ln 2} \times \theta_{\text {major }} \times \theta_{\text {minor }}$ is solid angle of the synthesized beam, $S_{\nu}^{b e a m}$ is the peak flux density measured in unit of mJy/beam, $\tau_{\nu}$ is optical depth, and $\mathrm{T}_{\mathrm{d}}$ is dust temperature and $\mathrm{B}_{\nu}\left(\mathrm{T}_{\mathrm{d}}\right)$ is Planck function (Whittet 1992). Optical depth can be expressed as,

$$
\tau_{\nu}=\rho_{d} \kappa_{\nu} L
$$

where $\rho_{d}$ is the mass density of dust, $\kappa_{\nu}$ is the mass absorption coefficient, and $\mathrm{L}$ is the path length. Using the dust to gas mass ratio $(Z)$, the mass density of the dust can be written as,

$$
\rho_{d}=Z \mu_{H} \rho_{H_{2}}=2 Z \mu_{H} N_{H_{2}} m_{H} / L
$$

where $\rho_{\mathrm{H}_{2}}$ is the mass density of hydrogen molecule, $\mathrm{N}_{\mathrm{H}_{2}}$ is the column density of hydrogen, $\mathrm{m}_{\mathrm{H}}$ is the hydrogen mass, and $\mu_{\mathrm{H}}$ is the mean atomic mass per hydrogen atom. Here, we used $Z=0.01, \mu_{H}=1.41$ (Cox et al. 2000), and dust temperature $150 \mathrm{~K}$. Two dimensional Gaussian fitting of the continuum yields a peak intensity $\left(\mathrm{S}_{\nu}^{\text {beam }}\right)$ of dust continuum of $158.4 \mathrm{mJy} / \mathrm{beam}$ and integrated flux $\left(\mathrm{F}_{\nu}\right)$ of $242.4 \mathrm{mJy}$. RMS noise of the continuum map was found to be $5 \mathrm{mJy} /$ beam. From the above equations column density of molecular hydrogen can be written as,

$$
N_{H_{2}}=\frac{S_{\nu}^{\text {beam }} / \Omega_{\text {beam }}}{2 \kappa_{\nu} B_{\nu}\left(T_{d}\right) Z \mu_{H} m_{H}} .
$$

According to the interpolation of the data presented in Ossenkopf \& Henning (1994), we estimated the mass absorption coefficient. By considering a thin ice condition, here, we obtained the mass absorption coefficient at $94 \mathrm{GHz}$ (3187.90 micron) $0.176 \mathrm{~cm}^{2} / \mathrm{g}$. By using equation 4 , total hydrogen column density of G31 source is $\sim 1.53 \times 10^{25} \mathrm{~cm}^{-2}$.

\subsection{Line analysis, molecular emission and spectral fitting}

Observed spectra are obtained within the circular region having a diameter of $1.1^{\prime \prime}$ centered at RA (J2000) $=18^{h} 47^{m} 34.309^{s}$, Dec $(\mathrm{J} 2000)=-1^{o} 12^{\prime} 46.00^{\prime \prime}$. Line parameters of all the observed transitions are obtained using a single Gaussian fit. From fitting, we have estimated the line width (FWHM), LSR velocity, and the integrated intensity. All the line parameters of observed molecules such as molecular transitions (quantum numbers) along with their rest frequency $(\nu)$, line width (FWHM, $\Delta V)$, integrated intensity $\left(\int \mathrm{T}_{\mathrm{mb}} \mathrm{dV}\right)$, upper state energy $\left(\mathrm{E}_{u}\right), \mathrm{V}_{\mathrm{LSR}}$, and transition line intensity $\left(\mathrm{S} \mu^{2}\right.$, where $\mathrm{S}$ is the transition line strength and $\mu$ is the dipole moment) are presented in Table 1. Overall, we have observed a broad line width $\left(\geq 5 \mathrm{~km} \mathrm{~s}^{-1}\right)$ and all lines are at the center around the systematic velocity of the source $\left(\sim 97 \mathrm{~km} \mathrm{~s}^{-1}\right)$ (see Table 1$)$. In the Appendix, we have presented observed and Gaussian fitted spectra (see Figures A2-A6).

\subsubsection{Simple Molecules}

We have observed $\mathrm{SO}_{2}(86.63908 \mathrm{GHz})$ and $\mathrm{H}_{2} \mathrm{CO}$ $(101.33299 \mathrm{GHz})$ in $\mathrm{G} 31$. We have also identified some simple molecules such as $\mathrm{HCN}, \mathrm{H}^{13} \mathrm{CO}^{+}$, and $\mathrm{SiO}$, which are widely used to trace various physical processes of star-forming regions. The observed spectral profile of $\mathrm{H}^{13} \mathrm{CO}^{+}$shows red-shifted absorption, which is shown in Figure 2a. This is a signature of inverse P-Cygni profile. This suggests that the source has an infall nature. Observed emission of $\mathrm{SiO}$ and $\mathrm{HCN}$ traces the outflows in this source.

Figure $2 \mathrm{~b}$ shows the observed spectral profile of HCN. Here, we have identified three hyperfine transitions of $\mathrm{HCN}(\mathrm{F}=1 \rightarrow 1,2 \rightarrow 1$, and $1 \rightarrow 0$ hyperfine components of the $\mathrm{J}=1 \rightarrow 0$ transition, see Table 2). Three hyperfine transitions of $\mathrm{HCN}$ are appeared in absorption and blended together. These three absorption lines together resulting in a broad feature. $\mathrm{HCN}$ traces the outflow associated with the source. The observed transitions of both $\mathrm{HCN}$ and $\mathrm{H}^{13} \mathrm{CO}^{+}$are found to be optically thick.

Here, we have also detected one transition of $\mathrm{SiO}$ at $86.84696 \mathrm{GHz}(\mathrm{J}=2 \rightarrow 1)$. $\mathrm{SiO}$ shows the absorption feature towards the center of the dust continuum and emission profile at all other positions. (Beltrán et al. 2018) also observed similar spectral feature of $\mathrm{SiO}$ (54, $217.10498 \mathrm{GHz}$ ). $\mathrm{SiO}$ traces outflows associated with this source.

\subsubsection{Complex Organic Molecules}

We have observed numerous COMs that were previously reported in G31 (e.g., Rivilla et al. 2017, and references therein). Additionally, for the first time in this source, we are reporting the detection of two new molecules, methyl isocyanate $\left(\mathrm{CH}_{3} \mathrm{NCO}\right)$ and methanethiol $\left(\mathrm{CH}_{3} \mathrm{SH}\right)$. Altogether, we have identified six transitions of $\mathrm{CH}_{3} \mathrm{NCO}$ with the ground vibrational state $\left(v_{b}=0\right)$ and one transition with $v_{b}=1$. Among the six transitions of $\mathrm{CH}_{3} \mathrm{NCO}$, one transition (86.86674 $\mathrm{GHz}$ ) is blended with ethylene glycol (confirmed from LTE modeling).

We have identified three different peaks of $\mathrm{CH}_{3} \mathrm{SH}$, where each peak contains multiple transitions. These transitions are reported in Table 1. We are unable to separate these observed transitions. Because of closely 

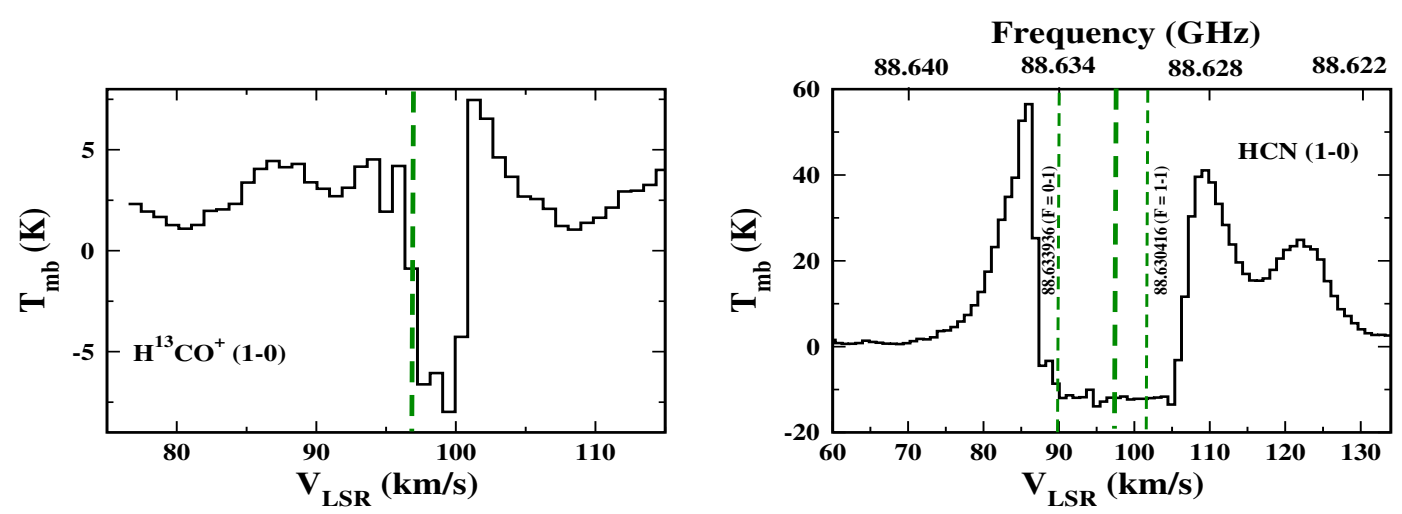

Figure 2. Observed spectra of (a) $\mathrm{H}^{13} \mathrm{CO}^{+}$(inverse P-Cygni) and (b) $\mathrm{HCN}$ towards the dust continuum peak. $86.631847 \mathrm{GHz}$ transition of $\mathrm{HCN}$ is used to set the velocity scale. Other two lines (green dashed) are plotted in the same figure by taking the difference in frequency between the transitions which corresponds to have velocity shift by $\sim 7 \mathrm{~km} \mathrm{~s}^{-1}$. for transition 88.63393 and $\sim 5 \mathrm{~km} \mathrm{~s}^{-1}$ for $88.63041 \mathrm{GHz}$. Bold dashed green line shows the systematic velocity $\left(\mathrm{V}_{\mathrm{LSR}}\right)$ of the source $\sim 97 \mathrm{~km} \mathrm{~s}$.

spaced transitions, it is difficult to distinguish two or more different transitions from a single observed peak of $\mathrm{CH}_{3} \mathrm{SH}$. Thus, to obtain the observed line parameters of $\mathrm{CH}_{3} \mathrm{SH}$ transitions, we have fitted multiple Gaussian components to the observed spectra. For multiple Gaussian fitting, we have used fixed values of velocity separation and expected line intensity ratio. Only FWHM is kept as a free parameter. This method works well in the observed spectral profile 1 and 3 around $101.139 \mathrm{GHz}$ and $101.167 \mathrm{GHz}$ of $\mathrm{CH}_{3} \mathrm{SH}$, where there are two transitions around each profile (see Table 1). Two-component Gaussian fitting corresponding to the two transitions are used to fit those spectral profiles (see Table 1 and Figure A5). However, this method does not work well for spectral profile 2, around $101.159 \mathrm{GHz}$, where four transitions are present. The four transitions can be classified into two classes based on their expected peak intensity (equivalent to $\mathrm{S} \mu^{2}$ ) and $\mathrm{E}_{u p}$. The expected intensity ratio between these four transitions is 1:0.58:0.58:0.58. We have used two-component Gaussian fitting corresponding to the two classes of transitions, one for 101.15933 $\mathrm{GHz}\left(\mathrm{S} \mu^{2}=4.96, \mathrm{E}_{u p}=31.26 \mathrm{~K}\right)$ transition and another component for the other three transitions, 101.15999 $\mathrm{GHz}, 101.16066 \mathrm{GHz}, 101.16069 \mathrm{GHz}\left(\mathrm{S} \mu^{2}=2.90, \mathrm{E}_{u p}\right.$ $=52.55 \mathrm{~K})$ around $101.159 \mathrm{GHz}$ line profile. For the fitting, we have used 1:1.74 (1.74 is the sum of intensity of three transitions) intensity ratio for these two components. Therefore, resultant Gaussian corresponding to the three transitions can be obtained via this method; as these transitions are equally intense and have similar excitation temperature, the area under Gaussian can be divided by three to get the contribution from each transition multiplets. We have used only one transition from these multiplets for the rotation diagram of $\mathrm{CH}_{3} \mathrm{SH}$ since the integrated area of all these three transitions are the same and have similar upper state energy $\left(\mathrm{E}_{u p}\right)$.
Therefore, these fitting results are used meaningfully to estimate rotational temperature using the rotation diagram method. Obtained line parameters are further used in the rotation diagram of $\mathrm{CH}_{3} \mathrm{SH}$. For the rotation diagram of $\mathrm{CH}_{3} \mathrm{SH}$, we use $101.13915 \mathrm{GHz}, 101.13965$ $\mathrm{GHz}, 101.15933 \mathrm{GHz}, 101.1599 \mathrm{GHz}, 101.16715 \mathrm{GHz}$, and $101.16830 \mathrm{GHz}$ transitions. Gaussian fits of $\mathrm{CH}_{3} \mathrm{SH}$ observed spectra are provided in Figure A5.

We also have identified the presence of two abundant COMs methanol and methyl formate in this source. Methanol is one of the most abundant COMs in starforming regions. Here, we have identified seven transitions of $\mathrm{CH}_{3} \mathrm{OH}$ with different upper state energy in the ALMA band 3 regions. We have also identified $\mathrm{CH}_{3} \mathrm{OCHO}$ transitions with a wide range of upper-level energies $(17-207 \mathrm{~K})$, which are listed in Table 1. The integrated intensity maps of some unblended transitions of $\mathrm{SO}_{2}, \mathrm{H}_{2} \mathrm{CO}, \mathrm{CH}_{3} \mathrm{SH}, \mathrm{CH}_{3} \mathrm{OH}, \mathrm{CH}_{3} \mathrm{OHCO}$, and $\mathrm{CH}_{3} \mathrm{NCO}$ are shown in Figures 3(a-f) where continuum is overlaid on the integrated intensity map, and all other maps correspond to different frequencies are given in the Appendix (see Figures A9-A12). Using local thermodynamic equilibrium modeling and comparing with the integrated intensities of observed molecular transitions, we found that various transitions of $\mathrm{CH}_{3} \mathrm{OH}, \mathrm{CH}_{3} \mathrm{NCO}$, and $\mathrm{CH}_{3} \mathrm{OCHO}$ are optically thin.

For example, in LTE and optically thin condition, the expected line ratio between the $87.01608 \mathrm{GHz}$ and $86.68019 \mathrm{GHz}$ transitions of $\mathrm{CH}_{3} \mathrm{NCO}$ is 1.6 while the observed intensity (brightness temperature) ratio is 1.44. This suggests that these two lines are optically thin. Similarly for $86.78078 \mathrm{GHz}$ and $86.68019 \mathrm{GHz}$ transitions of $\mathrm{CH}_{3} \mathrm{NCO}$, the observed intensity ratio between these two lines is 1.35 , and the expected line ratio is 1.30 . For the $101.12685 \mathrm{GHz}$ and $101.46980 \mathrm{GHz}$ transitions of $\mathrm{CH}_{3} \mathrm{OH}$, the observed intensity ratio is 2.3 , 

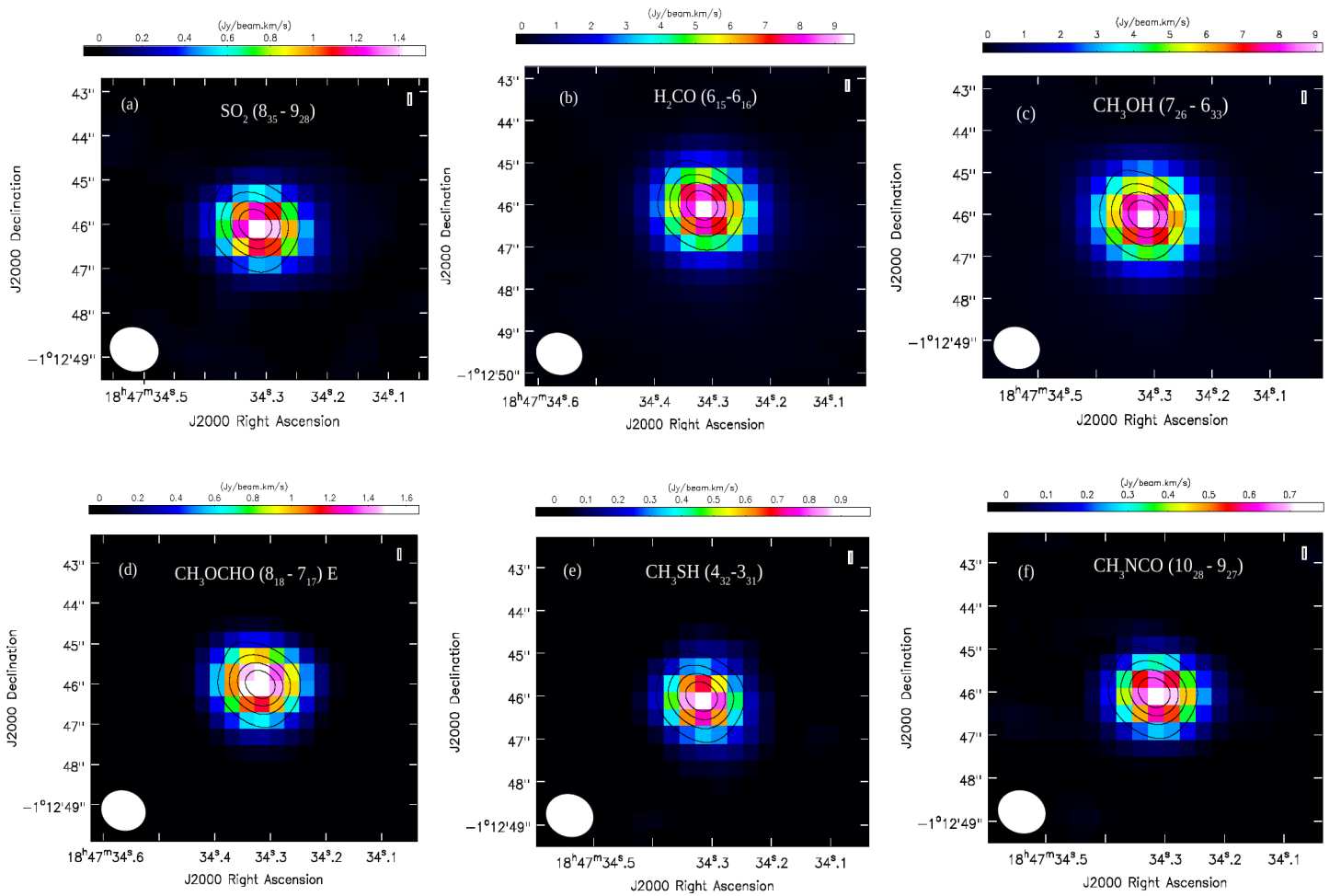

Figure 3. The integrated intensity distribution (color) of (a) $\mathrm{SO}_{2}$, (b) $\mathrm{H}_{2} \mathrm{CO}$, (c) $\mathrm{CH}_{3} \mathrm{OH}$, (d) $\mathrm{CH}_{3} \mathrm{OCHO},(e) \mathrm{CH}_{3} \mathrm{SH}_{\text {, (f) and }}$ $\mathrm{CH}_{3} \mathrm{NCO}$ lines overlaid of the $3.1 \mathrm{~mm}$ continuum emission (black contours) is shown. Contour levels are at $20 \%, 40 \%, 60 \%$, and $80 \%$ of the peak flux. The synthesized beam is shown in the lower left-hand corner of each figure.

which is consistent with the expected LTE line ratio of 2.2. For of $\mathrm{CH}_{3} \mathrm{OCHO}$, the observed line ratio between $88.68688 \mathrm{GHz}$ and $88.85160 \mathrm{GHz}$ transition is 4 , which is similar to the observed integrated intensity ratio 3.7. The similarity between the expected and observed line ratios of these transitions suggest that they are optically thin.

\subsection{Rotation diagram analysis: estimation of gas temperature}

We have detected multiple transitions of $\mathrm{CH}_{3} \mathrm{OH}$, $\mathrm{CH}_{3} \mathrm{OCHO}, \mathrm{CH}_{3} \mathrm{SH}$, and $\mathrm{CH}_{3} \mathrm{NCO}$, with different $\mathrm{E}_{\mathrm{u}}$. Thus, we employed the rotation diagram analysis to obtain the rotational temperatures. We performed the rotation diagram analysis by assuming the observed transitions to be optically thin and are in Local Thermodynamic Equilibrium (LTE). For optically thin lines, column density can be expressed as (Goldsmith \& Langer. 1999),

$$
\frac{N_{u}^{t h i n}}{g_{u}}=\frac{3 k_{B} \int T_{m b} d V}{8 \pi^{3} \nu S \mu^{2}},
$$

where $g_{u}$ is the degeneracy of the upper state, $k_{\mathrm{B}}$ is the Boltzmann constant, $\int \mathrm{T}_{\mathrm{mb}} \mathrm{dV}$ is the integrated intensity, $\nu$ is the rest frequency, $\mu$ is the electric dipole moment, and $\mathrm{S}$ is the transition line strength. Under
LTE conditions, the total column density can be written as,

$$
\frac{N_{u}^{\text {thin }}}{g_{u}}=\frac{N_{\text {total }}}{Q\left(T_{\text {rot }}\right)} \exp ^{-E_{u} / k_{B} T_{\text {rot }}},
$$

where $T_{\text {rot }}$ is the rotational temperature, $\mathrm{E}_{u}$ is the upper state energy, $\mathrm{Q}\left(\mathrm{T}_{\text {rot }}\right)$ is the partition function at rotational temperature. The equation 6 can be rearranged as,

$$
\log \left(\frac{N_{u}^{t h i n}}{g_{u}}\right)=-\left(\frac{\log e}{T_{\text {rot }}}\right)\left(\frac{E_{u}}{k_{B}}\right)+\log \left(\frac{N_{\text {total }}}{Q\left(T_{\text {rot }}\right)}\right) .
$$

Equation 7 shows that there is a linear relationship between $\mathrm{E}_{u}$ and $\log \left(\mathrm{N}_{\mathrm{u}} / \mathrm{g}_{\mathrm{u}}\right)$. Two parameters, column density and rotational temperature can be obtained by fitting a straight line to the values of $\log \left(\mathrm{N}_{\mathrm{u}} / \mathrm{g}_{\mathrm{u}}\right)$ plotted as a function of $\mathrm{E}_{u}$, where $\mathrm{N}_{\mathrm{u}} / \mathrm{g}_{\mathrm{u}}$ is obtained from the observations through equation 5 .

Rotational diagrams of methyl isocyanate, methanol, methanethiol, and methyl formate are presented in Figure 4. For $\mathrm{CH}_{3} \mathrm{OCHO}$, we have several transitions, but we do not see two components. Hence we fit all the data points with a line, which are not aligned. Though we did not find a very good fitting, the obtained rotational 
temperature of methyl formate $\sim 162 \mathrm{~K}$ is consistent with the previously measured value in this source for the same molecule (Beltrán et al. 2005; Rivilla et al. 2017). We have obtained a rotational temperature of 76 $\mathrm{K}$ for $\mathrm{CH}_{3} \mathrm{SH}$ and $48 \mathrm{~K}$ for $\mathrm{CH}_{3} \mathrm{NCO}$. The rotational diagram analysis suggests two temperature components of methanol, one is a cold component, and another is a hot component. Cold component (solid black line, see Figure $4 \mathrm{~b}$ ) temperature is obtained $33 \mathrm{~K}$. In contrast, hot component (solid blue line, see Figure 4b) temperature is $181 \mathrm{~K}$. Rotational diagram of $\mathrm{CH}_{3} \mathrm{OH}$ suggests that the transitions associated with the higher $\mathrm{E}_{\mathrm{u}}$ transitions (86.61557 GHz, 86.90291 GHz, 88.59478 GHz, 88.93997 $\mathrm{GHz}$; hot component) are mainly arising from the hot gas surrounding the G31. In contrast, the emissions associated with the lower $E_{u}(101.12685 \mathrm{GHz}, 101.29341$ $\mathrm{GHz}, 101.46980 \mathrm{GHz}$; cold component) transitions are from the cold region of the HMC. However, due to our observation's lower angular and spatial resolution, we marginally resolved these transitions. Therefore, it is not possible to provide a clear insight into the spatial distribution of methanol in this source.

The error bars (vertical red bars) in Figure 4 are the absolute uncertainty in a $\log$ of $\left(\mathrm{N}_{u} / \mathrm{g}_{u}\right)$, and this arises from the error of the observed integrated intensity that we measured using a single Gaussian fitting to the observed profile of each transition. The integrated intensities with their uncertainties of all observed transitions are provided in Table 1. Our estimated rotational temperatures for methyl formate and the hot component of methanol are similar to the typical hot core temperature. On the other hand, methyl isocyanate and the cold component of methanol have lower rotational temperatures (33-48 K). However, due to poor angular resolution, we could not provide further details about these two temperature components' spatial distribution.

To obtain the molecular column density of the observed chemical species, we used LTE modeling with CASSIS. For this modeling, we used the estimated rotational temperature discussed above. We calculated beam average column density $\left(\mathrm{N}_{b}\right)$, where the effect of beam dilution was not taken into consideration. For the model, we used five parameters; (i) column density, (ii) excitation temperature $\left(\mathrm{T}_{e x}\right)$, (iii) line width $(\Delta \mathrm{V})$, (iv) velocity $\left(\mathrm{V}_{L S R}\right)$, and $(\mathrm{v})$ source size $\left(\theta_{s}\right)$. To obtain the best fit with the observation, only column density was varied by keeping all other parameters (Tex, source size, FWHM, and hydrogen column density) fixed and we choose the best fitted-results when LTE model spectra are the same as the observed spectra. For LTE fitting, we used the source sizes for molecular emission similar to the deconvolved size as we obtained from 2D Gaussian fitting. Line width (FWHM) for molecular transitions are estimated from the Gaussian fitting of spectral profiles, and excitation temperature (Tex) is assumed to be $\sim 150 \mathrm{~K}$, a typical hot core temperature. Fixed parameters are noted in Table 3 . We did not have a rotation diagram for all the species (e.g., $\mathrm{SO}_{2}, \mathrm{H}_{2} \mathrm{CO}$ ) presented here. Thus, we followed the LTE model to derive the column density of all the species. We have also compared the column densities of $\mathrm{CH}_{3} \mathrm{OH}, \mathrm{CH}_{3} \mathrm{SH}, \mathrm{CH}_{3} \mathrm{NCO}$, and $\mathrm{CH}_{3} \mathrm{OCHO}$ between rotation diagram analysis and LTE model (see Table 4 and Section 3.6). Observed column densities and fractional abundances of all the species are presented in Table 4. Abundances of all species are noted w.r.to $\mathrm{H}_{2}$.

\subsection{Radiative transfer modeling}

Absorption studies are best-suited to trace the physical conditions (e.g., density, kinetic temperature) of the ISM because it is not induced by beam dilution as long as the absorbing layer is larger than the continuum source. Here, we used non-LTE modeling to derive excitation conditions and physical properties from these absorption features. For the non-LTE modeling, we use RADEX program (Van der Tak et al. 2007). The collisional rates were taken from LAMDA database and the spectroscopic constants were taken from JPL/CDMS catalog. In RADEX, we used the continuum background temperature $\left(\mathrm{T}_{c}\right)$ of $36 \mathrm{~K}$, which corresponds to the peak brightness temperature of the $3.2 \mathrm{~mm}$ continuum by considering the beam dilution. For this modeling, we have used colum density $3.55 \times 10^{15} \mathrm{~cm}^{-2}, 8.92 \times 10^{15} \mathrm{~cm}^{-2}$, and $1.18 \times 10^{15} \times \mathrm{cm}^{-2}$ for $\mathrm{H}^{13} \mathrm{CO}^{+}, \mathrm{HCN}$, and $\mathrm{SiO}$ respectively. Figures $5(\mathrm{a})$-(c) shows the variation of radiation temperature of $\mathrm{HCN}(1-0), \mathrm{H}^{13} \mathrm{CO}^{+}(1-0)$, and $\mathrm{SiO}(2-1)$ respectively for a wide range of parameter space. Our parameter space consists of the number density of hydrogen (varies in between $10^{3}-10^{7} \mathrm{~cm}^{-3}$ ) and kinetic temperature (varies in between 1-100 K). Figures 5(a)-(c) shows the parameter space for the absorption features of $\mathrm{HCN}, \mathrm{H}^{13} \mathrm{CO}^{+}$, and $\mathrm{SiO}$ respectively. We have estimated the critical density of HCN (1-0), $\mathrm{H}^{13} \mathrm{CO}^{+}(1-0)$, and $\mathrm{SiO}(2-1)$, which are $1.51 \times 10^{6} \mathrm{~cm}^{-3}$, $1.83 \times 10^{5} \mathrm{~cm}^{-3}$, and $2.26 \times 10^{5} \mathrm{~cm}^{-3}$ respectively. We have shown that for hydrogen density $\geq 1.0 \times 10^{5} \mathrm{~cm}^{-3}$, all these transitions are showing absorption features. If $\mathrm{H}_{2}$ density is below the critical density, level populations are mostly governed by collisional excitation and deexcitation rather than Boltzmann distribution. . Thus these molecules might show emission even at $\mathrm{T} \leq \mathrm{T}_{c}$. The observed spectral profile of $\mathrm{SiO}$ is in line with this results. We found $\mathrm{SiO}$ emission at all positions except the 
Methyl Isocyanate
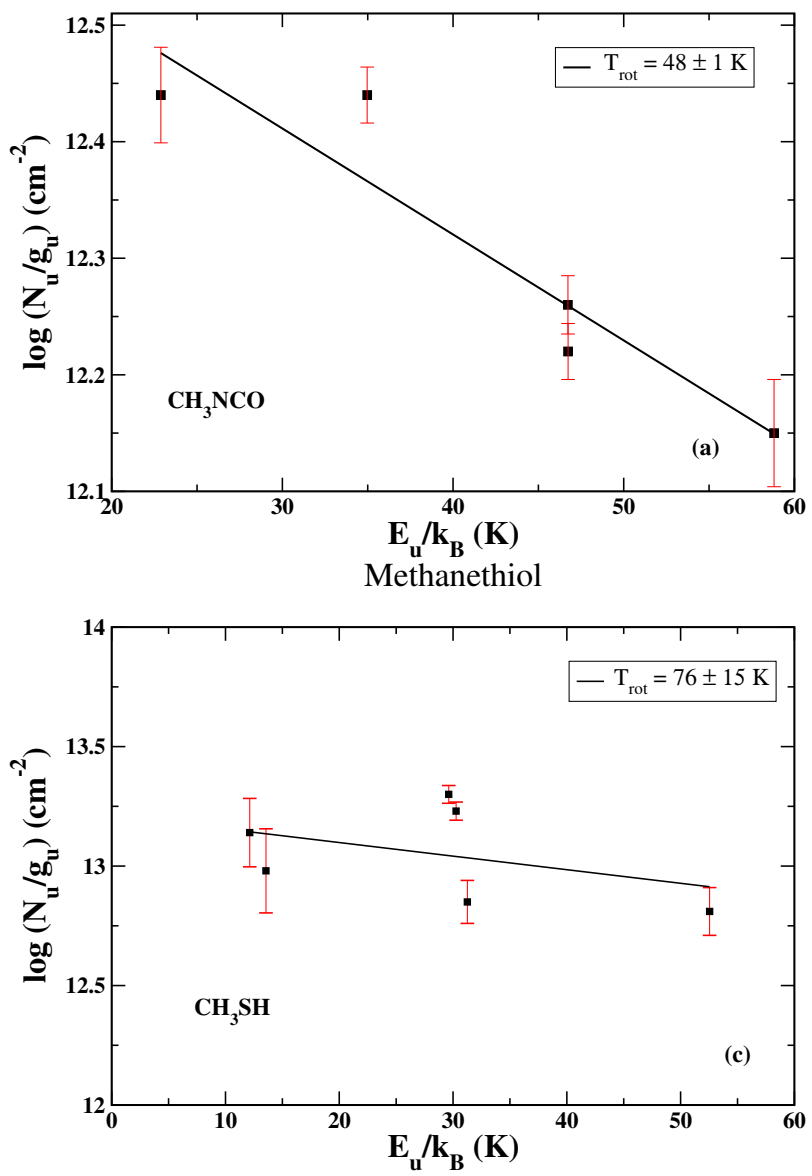

Methanol
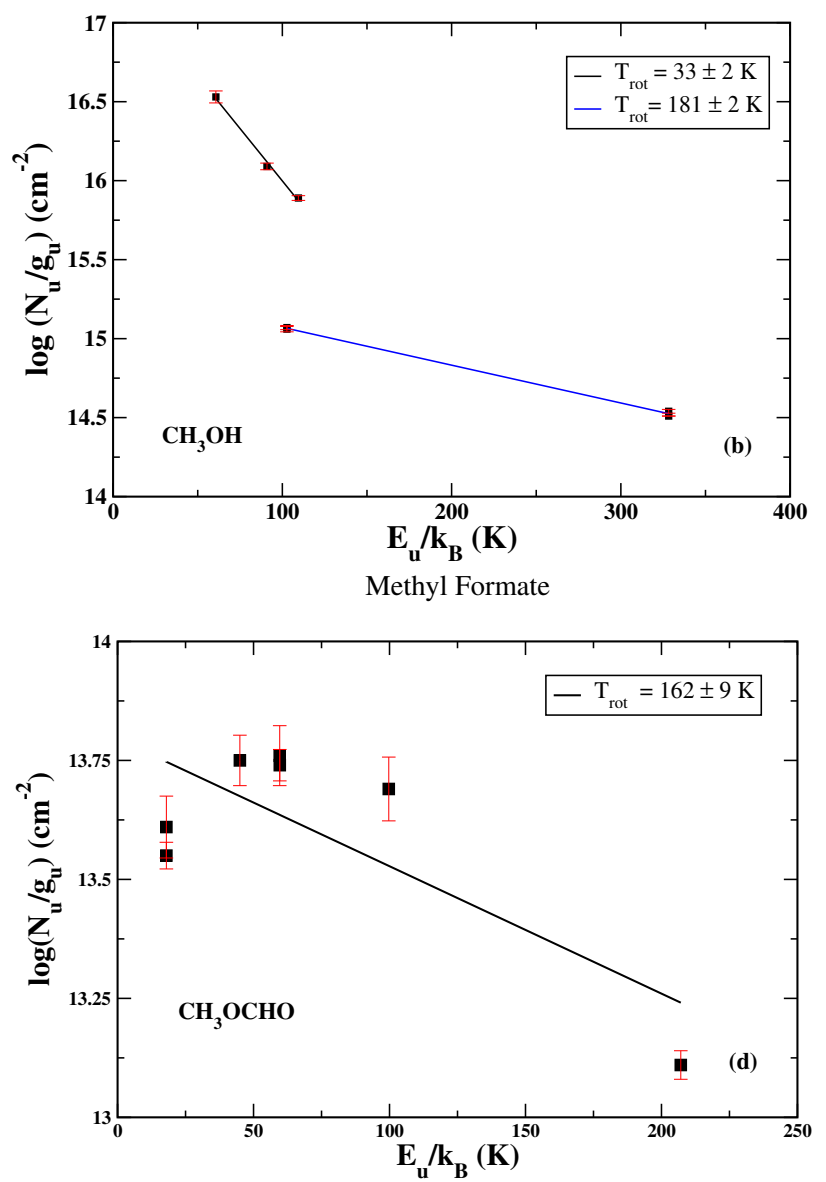

Figure 4. Rotational diagram of (a) $\mathrm{CH}_{3} \mathrm{NCO}$, (b) $\mathrm{CH}_{3} \mathrm{OH}$, (c) $\mathrm{CH}_{3} \mathrm{SH}$, and (d) $\mathrm{CH}_{3} \mathrm{OCHO}$. Black filled square shows the data points, and the solid black and blue lines represent the fitted curve.

source's central region (where density is high, which is probably greater than critical density).

\subsection{Physical properties of the source}

In this source, we obtained a temperature range 33 to $180 \mathrm{~K}$. Hydrogen column density of this source is estimated $\sim 1.53 \times 10^{25} \mathrm{~cm}^{-2}$. Various observed molecular emissions are discussed below.

\subsubsection{Infalling envelope}

Infall nature was observed in G31 earlier by various authors (Girart et al. 2009; Mayen-Gijon et al. 2014; Beltrán et al. 2018, e.g.,). Girart et al. (2009) observed an inverse P-Cygni profile in G31 with low energy lines of $\mathrm{C}^{34} \mathrm{~S}$ (7-6). Recently, Beltrán et al. (2018) identified red-shifted absorption of $\mathrm{H}_{2} \mathrm{CO}$ and $\mathrm{CH}_{3} \mathrm{CN}$ and its isotopologues towards the dust continuum of the main core with different upper state energy. Mayen-Gijon et al. (2014) observed various inversion transitions $(2,2)$, $(3,3),(4,4),(5,5)$ and $(6,6)$ of ammonia by using Very Large Array (VLA) observations to understand the infall motion in G31. To explain the infall motion in
G31, they used a different approach called the central blue spot which can be easily identified in the first-order moment maps. The central blue spot consists of blueshifted emission in the first-order moment towards the zeroth-order moment map's peak position. They found the central blue spot infall signature in all observed transitions of ammonia. In this work, we have detected one transition of $\mathrm{H}^{13} \mathrm{CO}^{+}$(1-0) at frequency $86.75430 \mathrm{GHz}$, which shows both absorption and emission nature (see Figure 2a). This is an inverse P-Cygni profile. The infall velocity can be estimated as the difference between systematic velocity and the velocity at the observed inverse P-Cygni profile's absorption feature. The infall velocity of the source is obtained to be $4.5 \mathrm{~km} \mathrm{~s}^{-1}$. This suggests that matter is infalling from the envelope towards the source's central region, which is consistent with the earlier observations (Girart et al. 2009; Beltrán et al. 2018).

\subsubsection{Molecular outflow}


Table 1. Summary of the line parameters of observed molecules towards G31.41+0.31.



NOTES: ${ }^{1}$ Spectral profile $1,{ }^{2}$ Spectral profile $2,{ }^{3}$ Spectral profile $3,{ }^{i}$ velocity with respect to $101.13915 \mathrm{GHz}$ transition, ${ }^{i i}$ velocity w.r.t $101.15933 \mathrm{GHz}$ transition, ${ }^{\text {iii }}$ velocity w.r.t $101.16715 \mathrm{GHz}$ transition.

Table 2. Observed molecular transitions in absorption.

\begin{tabular}{|c|c|c|c|}
\hline \hline Species & $\left(\mathrm{J}_{\left.\mathrm{K}_{\mathrm{a}}^{\prime} \mathrm{K}_{c}^{\prime}-\mathrm{J}_{\mathrm{K}_{\mathrm{a}}^{\prime \prime} \mathrm{K}_{c}^{\prime \prime}}^{\prime \prime}\right)}\right.$ & Frequency in $\mathrm{GHz}$ & $\mathrm{E}_{u}(\mathrm{~K})$ \\
\hline \hline $\mathrm{H}^{13} \mathrm{CO}^{+}$ & $1-0$ & 86.75430 & 4.16 \\
$\mathrm{HCN}$ & $1-0(\mathrm{~F}=1-1)$ & 88.63041 & 4.25 \\
$\mathrm{HCN}$ & $1-0(\mathrm{~F}=2-1)$ & 88.63184 & 4.25 \\
$\mathrm{HCN}$ & $1-0(\mathrm{~F}=0-1)$ & 88.63393 & 4.25 \\
\hline \hline
\end{tabular}

Many authors previously studied molecular outflows associated with G31 (e.g., Cesaroni et al. 2011; Olmi et al. 1996; Beltrán et al. 2018). SiO and ${ }^{13} \mathrm{CO}$ revealed the presence of two molecular outflows: One in the East-West (E-W) direction and another one in the North-South (N-S) direction. SiO is an excellent tracer of shock and molecular outflow associated with the starforming regions (e.g., Zapata et al. 2009; Leurini et al. 
(a)

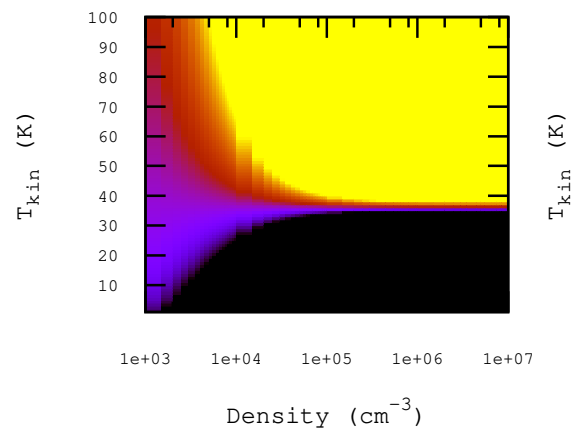

(b)

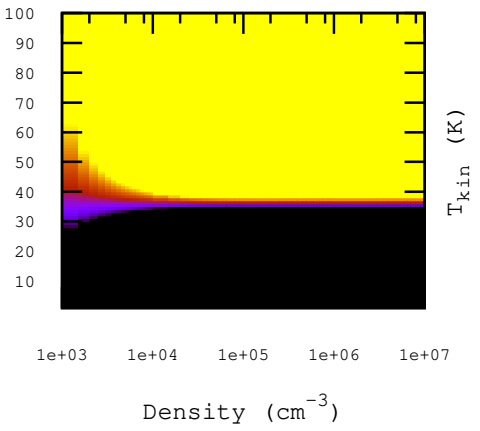

(c)

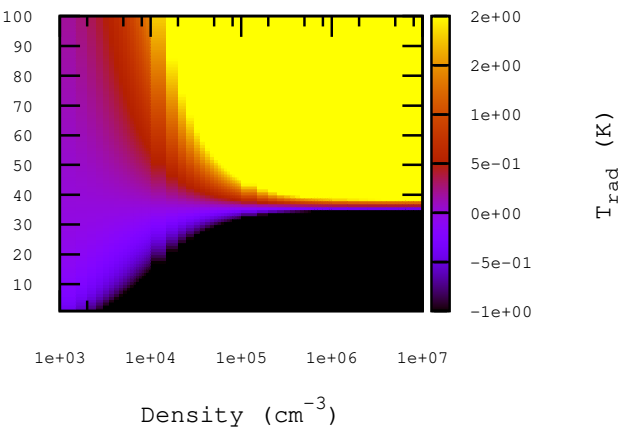

Figure 5. Variation of the radiation temperature of (a) $\mathrm{HCN}(1-0)$, (b) $\mathrm{H}^{13} \mathrm{CO}^{+}$(1-0), and (c) SiO (2-1) respectively for a wide range of parameter space. The vertical axis is the kinetic temperature, labeled as $\mathrm{T}_{\text {kin }}(\mathrm{K})$. The units of the radiation temperature $\left(\mathrm{T}_{\mathrm{rad}}\right)$ used in the color bar is in Kelvin.

Table 3. Input parameters of LTE model

\begin{tabular}{|c|c|c|}
\hline Species & Source size $\left(^{\prime \prime}\right)$ & FWHM $(\mathrm{km} / \mathrm{s})$ \\
\hline $\mathrm{SO}_{2}$ & 0.7 & 8.4 \\
$\mathrm{H}_{2} \mathrm{CO}$ & 1.3 & 10.2 \\
$\mathrm{CH}_{3} \mathrm{SH}$ & 0.8 & 5.5 \\
$\mathrm{CH}_{3} \mathrm{OH}$ & 1.3 & 8.5 \\
$\mathrm{CH}_{3} \mathrm{NCO}$ & 0.8 & 6.5 \\
$\mathrm{CH}_{3} \mathrm{OCHO}$ & 1.35 & 6.0 \\
$\mathrm{H}_{2}$ column density $=1.53 \times 10^{25} \mathrm{~cm}^{-2}$ \\
\multicolumn{2}{|c}{$\mathrm{T}_{e x}=150 \mathrm{~K}$}
\end{tabular}

2014). Beltrán et al. (2018) showed average red-shifted and blue-shifted wings of $\mathrm{SiO}$ emission, which reveals three outflow directions associated with this source: EW, N-S, and NE-SW. . Among these three outflow directions, N-S and E-W components are clearly present in our observation. However, NE-SW component is not very evident (see original Figure 7 and illustrated in Figure A1). Channel maps of $\mathrm{SiO}$ emission is shown in Figure 6. The blue-shifted and red-shifted integrated emission of $\mathrm{SiO}$ along with the continuum, is depicted in Figure 7. The presence of HCN is widespread in diverse interstellar environments ranging from dark cloud to star-forming region and circumstellar envelope of a carbon-rich star (e.g., Snyder \& Buhl. 1971; Ziurys \& Turner. 1986). HCN also traces molecular outflow in G31. The observed spectrum profile of HCN (see Figure $2 \mathrm{~b}$ ) is symmetric with respect to the systematic velocity of the source. However, the intensity of emission wings associated with the absorption profile is asymmetric. This may be due to the asymmetry in molecular distributions. Figure 8 shows the red-shifted and blue-shifted integrated emissions of $\mathrm{HCN}$ overlaid on the continuum.

To obtain the outflow parameters, we estimated the outflow velocity as the maximum velocity of outflow emission with respect to the systematic LSR velocity $\left(97 \mathrm{~km} \mathrm{~s}^{-1}\right)$. For the blue lobe and red lobe of $\mathrm{SiO}$ outflow, velocities are found to be $23 \mathrm{~km} \mathrm{~s}^{-1}$ and $33 \mathrm{~km}$ $\mathrm{s}^{-1}$. We estimated the dynamical timescale of outflow by using $\mathrm{t}_{d y n}=\frac{l(b, r)}{V_{\max }(b, r)}$. For this, we calculate two different lengths $(\mathrm{lb} 1, \mathrm{lb} 2)$ of the blue lobe and two lengths $(\operatorname{lr} 1, \operatorname{lr} 2)$ for red lobes (see Figure 7-8). We obtained the average dynamical time scale of outflow $\sim 4 \times 10^{3}$ years by considering the maximum velocity of $\mathrm{SiO}$ of the blue lobe and red lobe. The dynamical time scale of $\mathrm{HCN}$ outflow is obtained to be $\sim 3 \times 10^{3}$ years. These values are in good agreement with the dynamical time scale of outflow of ${ }^{12} \mathrm{CO}$ and $\mathrm{SiO}$, as previously reported in G31 by Cesaroni et al. (2011) and Beltrán et al. (2018). For all individual positions, the different dynamical time scales for both blue and red lobe are presented in Table 5. For HCN, we calculate the outflow parameters for one blue lobe (lb1) and one red lobe (lr1). To estimate the other outflow parameters such as mass, momentum, energy, mass loss, momentum loss, and energy loss per year, we used the formula mentioned in Cabrit \& Bertout. (1990). To estimate the outflow parameters, we assumed same abundance $\left(1.0 \times 10^{-8}\right)$ and excitation temperature $(20 \mathrm{~K})$ of $\mathrm{SiO}$ (Codella et al. 2013) and HCN. All the outflow parameters are summarized in Table 5. All these outflow parameters are in good agreement with the observed values of Araya et al. (2008).

In Figure 6, we have shown the channel maps of $\mathrm{SiO}$ emission associated with the HMC. This figure depicts that blue-shifted channels are distributed in the south- 
Table 4. Comparison of observed molecular column density, fractional abundances, and molecular ratios between G31 and other sources.

\begin{tabular}{|c|c|c|c|c|c|c|}
\hline \multirow[t]{2}{*}{ Species } & \multicolumn{2}{|c|}{$\begin{array}{c}\text { G31.41+0.31, } \\
\text { Column density } \\
\text { [Abundance] }\end{array}$} & \multirow[t]{2}{*}{$\begin{array}{c}\text { Sgr B2 }(\mathrm{N}) \\
\text { Column density } \\
\text { [Abundance] }\end{array}$} & \multirow[t]{2}{*}{$\begin{array}{c}\text { Orion KL } \\
\text { Column density } \\
\text { [Abundance] }\end{array}$} & \multirow[t]{2}{*}{$\begin{array}{c}\text { G10.47+0.03 } \\
\text { Column density } \\
\text { [Abundance] }\end{array}$} & \multirow[t]{2}{*}{$\begin{array}{c}\text { Chemical Model } \\
\text { Column density } \\
\text { [Abundance] }\end{array}$} \\
\hline & LTE & ation Diagram & & & & \\
\hline $\mathrm{CH}_{3} \mathrm{OH}$ & $1.84(19)[1.2(-6)]^{*}$ & $2.94(19)[1.92(-6)]$ & $4.00(19)[1.14(-5)]^{a}$ & $3.24(18)\left[1.62(-6]^{f}\right.$ & $9.0(18)[1.8(-6)]^{m}$ & $7.56(19)[2.10(-5)]^{n}$ \\
\hline $\mathrm{CH}_{3} \mathrm{OCHO}$ & $6.50(18)[4.25(-7)]$ & $4.30(18)[2.81(-7)]$ & $1.20(18)[3.33(-7)]^{b}$ & $1.04(17)[5.2(-8)]^{g}$ & $7.0(17)[1.4(-7)]^{m}$ & $3.31(17)[9.20(-8)]^{n}$ \\
\hline $\mathrm{CH}_{3} \mathrm{NCO}$ & $7.22(17)[4.72(-8)]^{*}$ & $1.58(16)[1.04(-9)]$ & $2.20(17)[6.11(-8)]^{c}$ & $7.0(15)[3.5(-9)]^{h}$ & $1.2(17)[8.88(-9)]^{o}$ & $4.68(16)[1.30(-8)]^{b}$ \\
\hline $\mathrm{CH}_{3} \mathrm{SH}$ & $4.13(17)[2.70(-8)]$ & $2.85(16)[1.86(-9)]$ & $3.40(17)[9.44(-8)]^{a}$ & $2.0(15)[1.0(-9)]^{i}$ & - & $1.33(16)[3.70(-9)]^{a}$ \\
\hline $\mathrm{SO}_{2}$ & $2.88(18)[1.88(-7)]$ & & $8.28(16)[2.30(-8)]^{d}$ & $1.0(17)[5.0(-8)]^{j}$ & $3.0(17)[6.0(-8)]^{m}$ & \\
\hline $\mathrm{H}_{2} \mathrm{CO}$ & $2.90(18)[1.90(-7)]$ & & $1.82(17)[5.06(-8)]^{e}$ & $1.5(17)[7.5(-8)]^{k}$ & $3.0(18)[6.0(-7)]^{m}$ & \\
\hline $\mathrm{SiO}$ & $1.46(15)[9.54(-11)]^{* *}$ & & $4.32(15)[1.20(-9)]^{d}$ & $5.4(14)[2.7(-10)]^{l}$ & - & \\
\hline $\mathrm{CH}_{3} \mathrm{SH} / \mathrm{CH}_{3} \mathrm{OH}$ & 0.0225 & 0.0010 & 0.0082 & 0.0006 & & 0.0002 \\
\hline $\mathrm{CH}_{3} \mathrm{OCHO} / \mathrm{CH}_{3} \mathrm{OH}$ & 0.3541 & 0.1463 & 0.0292 & 0.0321 & 0.0778 & 0.0044 \\
\hline $\mathrm{CH}_{3} \mathrm{NCO} / \mathrm{CH}_{3} \mathrm{OH}$ & 0.0393 & 0.0005 & 0.0054 & 0.0022 & 0.0049 & 0.0006 \\
\hline
\end{tabular}

${ }^{a}$ Müller et al. (2016), ${ }^{b}$ Belloche et al. (2016), ${ }^{c}$ Belloche et al. (2017), ${ }^{d}$ Higuchi et al. (2015), ${ }^{e}$ Belloche et al. (2013), ${ }^{f}$ Favre et al. (2015), ${ }^{g}$ Favre et al. (2011), ${ }^{h}$ Cernicharo et al. (2016), ${ }^{i}$ Kolesniková et al. (2014), ${ }^{j}$ Tercero et al. (2018), ${ }^{k}$ Esplugues et al. (2013), ${ }^{l}$ Schilke et al. (2001), ${ }^{m}$ Rolffs et al. (2011), ${ }^{n}$ Garrod et al. (2013), ${ }^{\circ}$ Gorai et al. (2020)

Orion $\mathrm{KL}\left(\mathrm{N}_{\mathrm{H}_{2}}\right)=2.0 \times 10^{24} \mathrm{~cm}^{-2}, \mathrm{Sgr} \mathrm{B} 2(\mathrm{~N})\left(\mathrm{N}_{\mathrm{H}_{2}}\right)=3.6 \times 10^{24} \mathrm{~cm}^{-2}, \mathrm{G} 10.47+0.03\left(\mathrm{~N}_{\mathrm{H}_{2}}\right)=5.0 \times 10^{24} \mathrm{~cm}^{-2}, \mathrm{G} 31.41+0.31\left(\mathrm{~N}_{\mathrm{H}_{2}}\right)=$ $1.53 \times 10^{25} \mathrm{~cm}^{-2}$

* For hot component of methanol, column density is derived using higher value of excitation temperature $\mathrm{T}=300 \mathrm{~K}$. Using LTE, we have found opacities $\geqslant 1$ for a few transitions of $\mathrm{CH}_{3} \mathrm{OH}(86.68655 \mathrm{GHz}, 86.68019 \mathrm{GHz}, 86.78077 \mathrm{GHz}$, and $86.80502 \mathrm{GHz})$ and $\mathrm{CH} 3 \mathrm{NCO}(86.68019 \mathrm{GHz}$ and $86.68655 \mathrm{GHz}$ ). Thus, the column density estimated for $\mathrm{CH}_{3} \mathrm{OH}$ and $\mathrm{CH}_{3} \mathrm{NCO}$ by using LTE should be considered as lower limit.

** $\mathrm{SiO}$ column density and fractional abundance calculated in the outflowing gas measured at $\mathrm{V}_{L S R}=94.1 \mathrm{~km} \mathrm{~s}^{-1}$ by averaging over the box $\left(7^{\prime \prime} \times 7^{\prime \prime}\right)$ centered at $(\alpha(\mathrm{J} 2000), \delta(\mathrm{J} 2000))=\left[18^{h} 47^{m} 34.121^{s},-1^{\circ} 12^{\prime} 48.975^{\prime \prime}\right]$.

west region and red-shifted channels toward the southeast and north-east regions. Bipolar lobe morphology observed from $93.2 \mathrm{~km} \mathrm{~s}^{-1}$ and $103.1 \mathrm{~km} \mathrm{~s}^{-1}$ channels suggest wide-angle bipolar outflow. Integrated emission of $\mathrm{SiO}$ and HCN transition (Figures 7 and 8) shows that there is a clear outflow direction along the East-West direction. In the integrated emission, the red lobe of outflow is slightly tilted to the south-east. This may occur due to another outflow direction along the southwestnortheast direction. There is also an outflow in the N-S direction. Outflow of $\mathrm{HCN}$ along the N-S direction is wider (see Figure 8) as compared to the $\mathrm{SiO}$ outflow (see Figure 7). This may be attributed to the excitation conditions and chemical origin of HCN. The estimated outflow is massive, $50 \mathrm{M}_{\odot}$ for $\mathrm{SiO}$ and $67 \mathrm{M}_{\odot}$ for $\mathrm{HCN}$ and the outflow is characterized as young $\left(3-4 \times 10^{3}\right.$ years). However, by considering the new distance measurement of $\sim 3.7 \mathrm{kpc}$ of this source, we obtained the dynamical time scale of outflow around $1-2 \times 10^{3}$ years. The estimated outflow mass is $\sim 11 \mathrm{M}_{\odot}$ for $\mathrm{SiO}$ and $\sim 14.6 \mathrm{M}_{\odot}$ for HCN.

\subsection{Molecular distributions and abundances}

In this section, we present the molecular abundance of all the observed species. We compare the abundance of all detected species between G31 and other sources, which is presented in Table 4. All the molecular abundances are reported w.r.t. $\mathrm{H}_{2}$. Figure A1 in the Appendix shows the schematic diagram of kinematics and molecular distributions of G31 based on present observation. There are three outflow directions one is along the $\mathrm{E}-\mathrm{W}$ direction, one is along the N-S direction, and another is along the NE-SW direction (only for $\mathrm{SiO}$ ). Outflow components are traced by $\mathrm{HCN}$ and $\mathrm{SiO}$ emissions. Here, we have obtained different molecular distributions of COMs. The emitting regions of different transitions of COMs are different. We have separated those into three regions such as the emitting region is smaller than the beam size $\left(\theta \sim 0.8^{\prime \prime}\right.$, see the purple circle in the Figure A1), comparable to the beam size $\left(\theta \sim 1.1^{\prime \prime}\right.$, see green circle outer edge in the Figure A1), and greater than the beam size $\left(\theta \sim 1.4^{\prime \prime}\right.$, see cyano circle in the Figure A1). Emitting region of all observed species are provided in Table B1 (see in Appendix). The uncertainties of emitting regions listed in Table B1 are the lower limit of true values as estimated emitting regions might be affected by several issues such as poor angular resolution, image noise, and non-Gaussian shape of the source. Emitting regions of $\mathrm{CH}_{3} \mathrm{OH}$ and $\mathrm{CH}_{3} \mathrm{OCHO}$ are comparable or slightly greater as compared to the beam size. Molecular distributions show that $\mathrm{CH}_{3} \mathrm{NCO}$ and $\mathrm{CH}_{3} \mathrm{SH}$ have a slightly lower-emitting region as compared to beam size (marginally resolved). The data presented in this paper suggests possibly different emitting regions for different COMs, but these are not conclusive because of comparable beam and source size. High sensitivity data with high angular and spatial resolution is required to understand the spatial distributions of these molecules. 


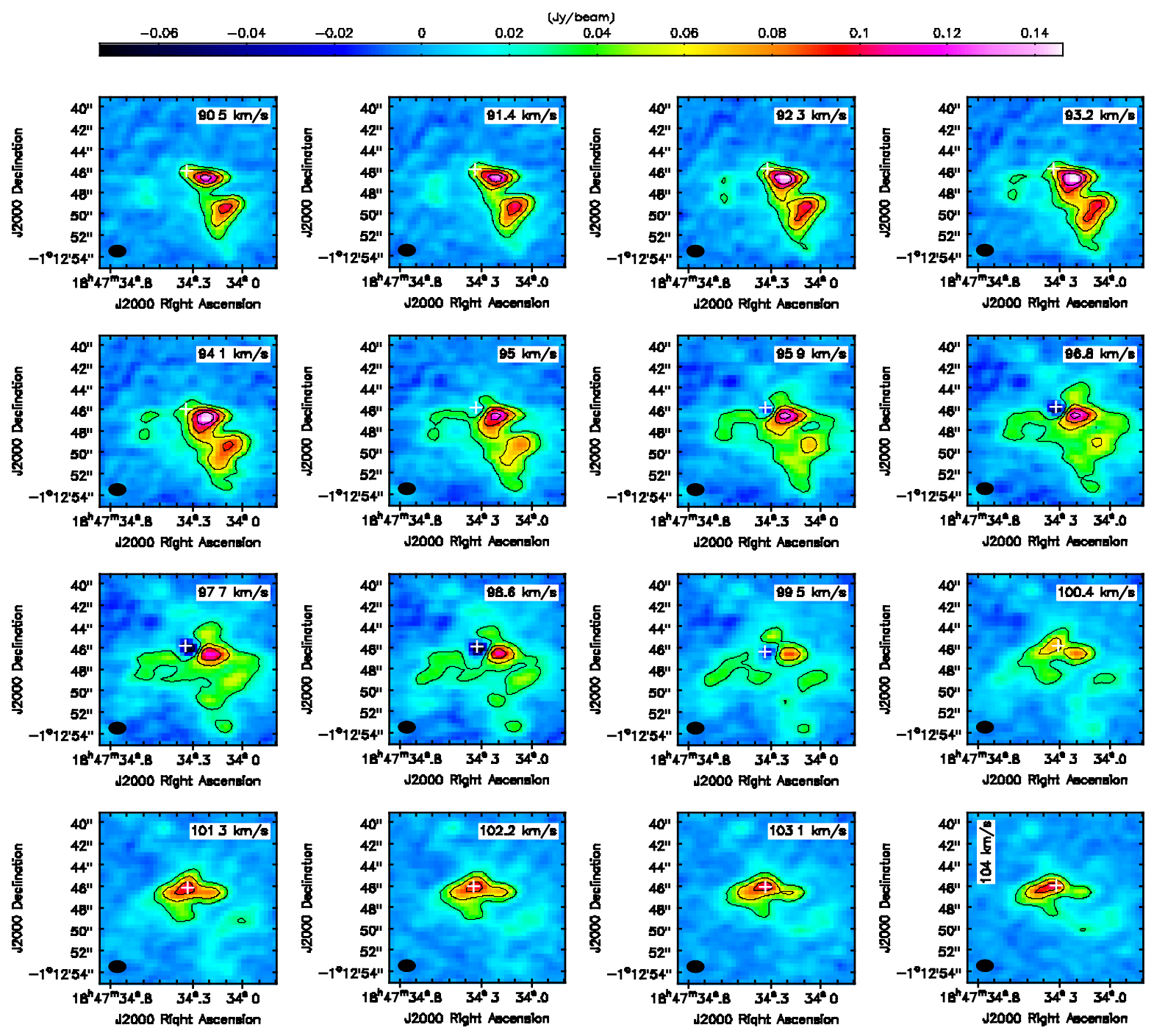

Figure 6. Channel maps of $\mathrm{SiO}(2-1)$ emission. Ellipse at the below left corner of the image shows the beam size $1.52^{\prime \prime} \times 1.09^{\prime \prime}$ with $\mathrm{PA}=88.98^{\circ}$. White plus sign indicates the position of the continuum.

\subsubsection{Sulfur dioxide, $\mathrm{SO}_{2}$}

$\mathrm{SO}_{2}$ is a good tracer of dense and hot gas of typical hot core (e.g., Charnley et al. 1997; Leurini et al. 2007). In this source, we detected a strong transition $\left(8_{3,5}-9_{2,8}\right)$ of $\mathrm{SO}_{2}$. Using LTE model, we estimated the column density and the fractional abundance of $\mathrm{SO}_{2}$, which are $\sim 2.88 \times 10^{18} \mathrm{~cm}^{-2}$ and $1.88 \times 10^{-7}$. The observed abundance of $\mathrm{SO}_{2}$ in G31 is one order of magnitude higher as compared to Srg B2, Orion KL and G10.47+0.03.

\subsubsection{Formaldehyde, $\mathrm{H}_{2} \mathrm{CO}$}

The presence of $\mathrm{H}_{2} \mathrm{CO}$ with its ortho, para, and isotopic form within the frequency range of $218-364$ $\mathrm{GHz}$ was previously reported by Isokoski, Bottinelli \& van Dishoeck. (2013). Red-shifted strong absorption of $\mathrm{H}_{2} \mathrm{CO}\left(\mathrm{E}_{u p}=21 \mathrm{~K}, 68 \mathrm{~K}\right)$ had recently been observed in G31, which supports the existence of infall in the core (Beltrán et al. 2018). Previously, Di Francesco et al.
(2001) reported a similar kind of inverse P-Cygni profile and found that $\mathrm{H}_{2} \mathrm{CO}$ traces infall in IRAS $4 \mathrm{~A}$ and they also detected the outflow from both $4 \mathrm{~A}$ and $4 \mathrm{~B}$ position by observing bright wing emission of $\mathrm{H}_{2} \mathrm{CO}$. Formaldehyde, along with its deuterated counterpart, has also been observed in outflows toward other sources (e.g., Sahu et al. 2018). The presence of $\mathrm{H}_{2} \mathrm{CS}$ in G31 has previously been reported by Ligterink et al. (2015). Here, we also have detected one strong emission line of $\mathrm{H}_{2} \mathrm{CS}$ at $101.478 \mathrm{GHz}$. However, the observed spectrum of $\mathrm{H}_{2} \mathrm{CS}$ shows that there is a blending with other molecular lines (methyl formate, confirmed from LTE modeling). " $\mathrm{H}_{2} \mathrm{CO}$ and $\mathrm{H}_{2} \mathrm{CS}$ are ubiquitous in the ISM. These two species have similar chemical structures; just $\mathrm{O}$ atom of $\mathrm{H}_{2} \mathrm{CO}$ is replaced by $\mathrm{S}$ atom in $\mathrm{H}_{2} \mathrm{CS}$.

\subsubsection{Methanol, $\mathrm{CH}_{3} \mathrm{OH}$}


Table 5. Physical parameters of the outflow

\begin{tabular}{|c|c|c|}
\hline Parameters & $\mathrm{SiO}$ & $\mathrm{HCN}$ \\
\hline \multicolumn{3}{|l|}{$\begin{array}{l}\text { Distance from continuum posi- } \\
\text { tion to outer contour }(\mathrm{pc})\end{array}$} \\
\hline Red lobe (lr1) & $0.117(0.055)$ & $0.118(0.055)$ \\
\hline Red lobe (lr2) & $0.087(0.041)$ & - \\
\hline Blue lobe1 (lb1) & $0.108(0.051)$ & $0.09(0.042)$ \\
\hline Blue lobe2 (lb2) & $0.135(0.063)$ & - \\
\hline \multicolumn{3}{|l|}{$\begin{array}{l}\text { Typical outflow } \quad \text { velocity: } \\
\mathrm{V}_{\text {outflow }}\left(\mathrm{km} \mathrm{s}^{-1}\right)\end{array}$} \\
\hline Red lobe & 33.0 & 40.0 \\
\hline Blue lobe & 23.0 & 30.0 \\
\hline \multicolumn{3}{|l|}{ Dynamical time: $\mathrm{t}_{d y n}(\mathrm{yr})$} \\
\hline \multirow[t]{2}{*}{ Blue lobe } & $4.59 \times 10^{3}\left(1.62 \times 10^{3}\right)$ & $2.87 \times 10^{3}\left(1.35 \times 10^{3}\right)$ \\
\hline & $5.74 \times 10^{3}\left(1.21 \times 10^{3}\right)$ & - \\
\hline \multirow[t]{2}{*}{ Red lobe } & $3.47 \times 10^{3}\left(2.16 \times 10^{3}\right)$ & $2.90 \times 10^{3}\left(1.36 \times 10^{3}\right)$ \\
\hline & $2.58 \times 10^{3}\left(2.70 \times 10^{3}\right)$ & - \\
\hline Average & $4.10 \times 10^{3}\left(1.92 \times 10^{3}\right)$ & $2.88 \times 10^{3}\left(1.36 \times 10^{3}\right)$ \\
\hline \multicolumn{3}{|l|}{ Outflow mass $\left(\mathrm{M}_{\odot}\right)$} \\
\hline Blue lobe & $27(6)$ & $12(2.6)$ \\
\hline Red lobe & $24(5)$ & $55(12)$ \\
\hline Total & $51(11)$ & $67(14.6)$ \\
\hline \multicolumn{3}{|l|}{ Momentum $\left(\mathrm{M}_{\odot} \mathrm{km} \mathrm{s}^{-1}\right)$} \\
\hline Blue lobe & $619(136)$ & $352(77)$ \\
\hline Red lobe & $790(173)$ & $2213(485)$ \\
\hline Total & 1409 (309) & $2565(562)$ \\
\hline \multicolumn{3}{|l|}{ Kinetic energy $\left(L_{\odot}\right.$ yr) } \\
\hline Blue lobe & $7.1 \times 10^{3}\left(1.6 \times 10^{3}\right)$ & $5.3 \times 10^{3}\left(1.2 \times 10^{3}\right)$ \\
\hline Red lobe & $1.3 \times 10^{4}\left(8.8 \times 10^{3}\right)$ & $4.4 \times 10^{4}\left(9.7 \times 10^{3}\right)$ \\
\hline Total & $2.0 \times 10^{4}\left(1.0 \times 10^{4}\right)$ & $4.9 \times 10^{4}\left(1.1 \times 10^{4}\right)$ \\
\hline \multicolumn{3}{|l|}{ Mass loss $\left(\mathrm{M}_{\odot} \mathrm{yr}^{-1}\right)$} \\
\hline Blue lobe & $5.2 \times 10^{-3}\left(4.1 \times 10^{-3}\right)$ & $4.1 \times 10^{-3}\left(1.9 \times 10^{-3}\right)$ \\
\hline Red lobe & $7.9 \times 10^{-3}\left(2.1 \times 10^{-3}\right)$ & $1.9 \times 10^{-2}\left(8.9 \times 10^{-3}\right)$ \\
\hline Total & $1.3 \times 10^{-2}\left(6.2 \times 10^{-3}\right)$ & $2.3 \times 10^{-2}\left(10.8 \times 10^{-3}\right)$ \\
\hline \multicolumn{3}{|l|}{$\begin{array}{l}\text { Momentum loss }\left(\mathrm{M}_{\odot} \mathrm{km} \mathrm{s}^{-1}\right. \\
\left.\mathrm{yr}^{-1}\right)\end{array}$} \\
\hline Blue lobe & $0.12(0.09)$ & $0.12(0.06)$ \\
\hline Red lobe & $0.26(0.07)$ & $0.76(0.35)$ \\
\hline Total & $0.38(0.16)$ & $0.88(0.41)$ \\
\hline \multicolumn{3}{|l|}{ Energy loss $\left(\mathrm{L}_{\odot}\right)$} \\
\hline Blue lobe & $1.38(1.10)$ & $1.84(0.85)$ \\
\hline Red lobe & $4.31(3.63)$ & $15.26(7.14)$ \\
\hline Total & $5.69(4.73)$ & $17.10(7.99)$ \\
\hline
\end{tabular}

Note: To estimate the $\mathrm{SiO}$ outflow physical parameters, we considered average value of red lobe $\left(\mathrm{r}_{r}=\frac{l r 1+l r 2}{2}\right)$ and blue lobe $\left(\mathrm{r}_{b}=\frac{l b 1+l b 2}{2}\right)$ and their corresponding average time scale of blue lobe and red lobe. We have calculated the lobe distance in arcsec and then convert into parsec by considering source distance $7.9 \mathrm{kpc}$. In parenthesis, we have noted the outflows parameters by considering the new distance $3.7 \mathrm{kpc}$ of G31.

Methanol is a well-known and one of the most abundant COMs in hot cores. Here, we have identified several transitions of methanol. LTE model suggests that it has a high fractional abundance $\sim 1.2 \times 10^{-6}$. We have also estimated methanol abundance following rotation diagram analysis. The obtained abundance using rotation diagram analysis is $\sim 1.2 \times 10^{-6}$, which is similar to the estimated results using the LTE model. A comparison of fractional abundance of methanol between G31 and other hot molecular core is presented in Table 4.

\subsubsection{Methanethiol, $\mathrm{CH}_{3} \mathrm{SH}$}

Methanethiol has a similar chemical structure as methanol; just $\mathrm{O}$ atom of methanol is replaced by $\mathrm{S}$ atom of methanethiol. In Figure A7, we show the observed and model (LTE) spectra of $\mathrm{CH}_{3} \mathrm{SH}$. Best fitted abundance and column density of methanethiol are $\sim 2.70 \times 10^{-8}$ and $\sim 4.13 \times 10^{-17}$ respectively. We also estimated the column density and fractional abundance of this species using rotation diagram analysis, which is one order of magnitude lower as compared to LTE model fitted results. The observed emitting region of methanethiol is comparable to the beam size. The spatial distribution of its emission is as compact as the dust continuum. Since our beam size is comparable to the source size. Therefore, we have uncertainties in determining the emitting regions of this species. For detail 


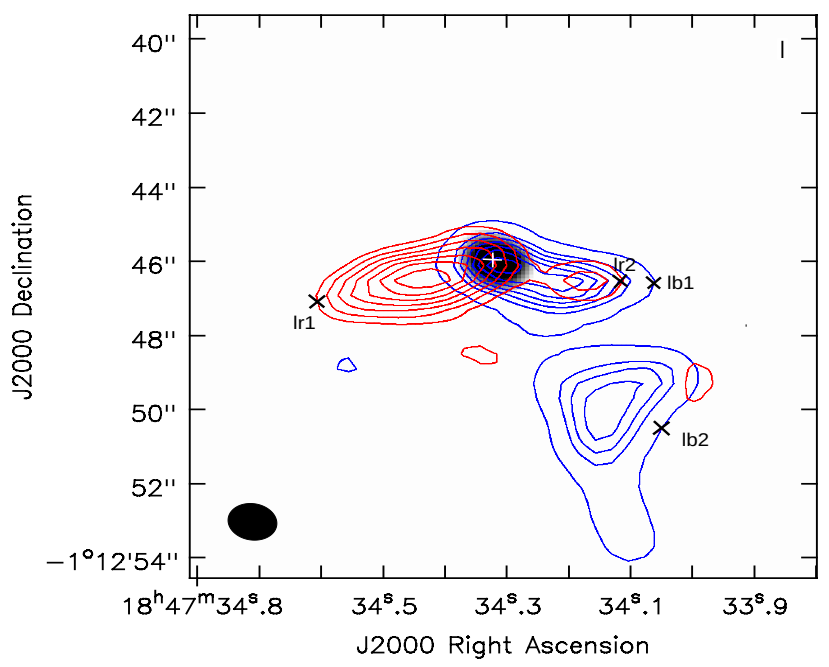

Figure 7. Gray scale levels for the continuum at $94 \mathrm{GHz}$. Blue-shifted emission of $\mathrm{SiO}$ integrated over $77-93 \mathrm{~km} \mathrm{~s}^{-1}$ where contour levels are at the 20,30, 40, 50,60, 70, $80 \%$ of the peak intensity. Red-shifted emission of $\mathrm{SiO}$ is integrated over $100-123 \mathrm{~km} \mathrm{~s}^{-1}$. Ellipse at the below left corner of the image shows the synthesized beam $\left(1.19^{\prime \prime} \times 0.98^{\prime \prime}\right)$. Plus sign indicates the position of the continuum. Two cross sign at the outer contour of red lobes indicates $\operatorname{lr} 1$ and $\operatorname{lr} 2$ distance another two cross sign on the red lobes indicated lb1 and lb2 distance.

knowledge about the distribution of this species, we need a high angular and spatial resolution data.

\subsubsection{Methyl formate, $\mathrm{CH}_{3} \mathrm{OCHO}$}

$\mathrm{CH}_{3} \mathrm{OCHO}$ is one of the most abundant COMs, which was observed in both low-mass and high-mass starforming regions (e.g., Brown et al. 1975; Cazaux et al. 2003). Previously, numerous transitions of $\mathrm{CH}_{3} \mathrm{OCHO}$ were identified in G31 (Isokoski, Bottinelli \& van Dishoeck. 2013; Rivilla et al. 2017; Beltrán et al. 2018). Column density, fractional abundance, and a comparison of fractional abundance of methyl formate with other hot cores are provided in Table 4 . The estimated column density and fractional abundance of methyl formate are similar using both rotation diagram and LTE model fitting analysis. Emitting diameters of various transitions of $\mathrm{CH}_{3} \mathrm{OCHO}$ are noted down in Table B1.

\subsubsection{Methyl isocyanate, $\mathrm{CH}_{3} \mathrm{NCO}$}

In the case of $\mathrm{CH}_{3} \mathrm{NCO}$, we have obtained a lower value of rotational temperature (48 K, see Figure. 4a), which is low as compared to a typical hot core. Methyl

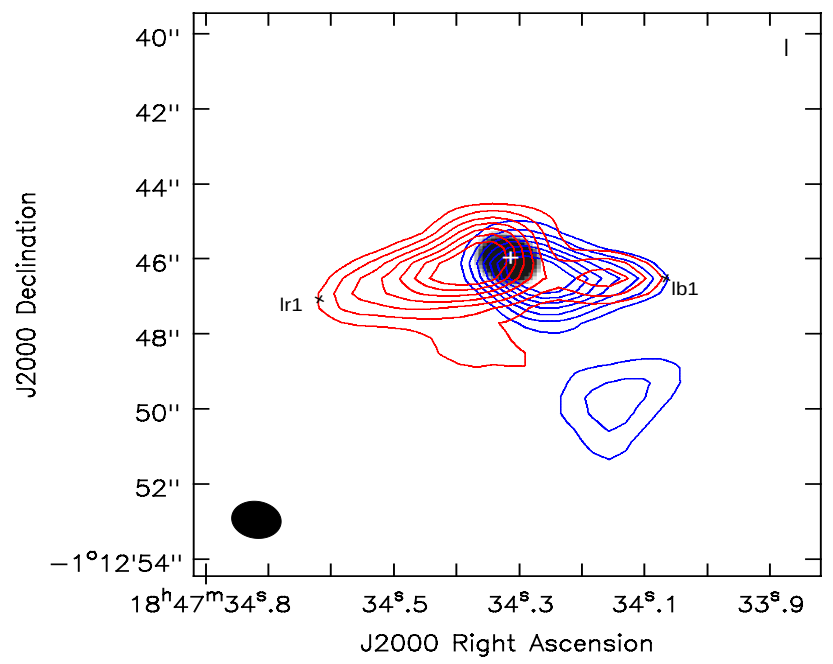

Figure 8. Gray scale levels are used for the continuum at $94 \mathrm{GHz}$. Blue-shifted emission of HCN integrated over 77-90 $\mathrm{km} \mathrm{s}^{-1}$ contours levels are at the 20, 30, 40, 50,60, 70, and $80 \%$ of the peak intensity. Red-shifted emission of HCN integrated over $105-115.7 \mathrm{~km} \mathrm{~s}^{-1}$. Ellipse at the below left corner of the image shows the synthesized beam $\left(1.19^{\prime \prime} \times 0.98^{\prime \prime}\right)$ with $\mathrm{PA}=76.83^{\circ}$

isocyanate transitions are compact and yield lower value rotational temperature. A similar trend has also been observed for cold component of methanol. Figure A8 shows the observed and model (LTE) spectra of $\mathrm{CH}_{3} \mathrm{NCO}$. The LTE model suggests that for a column density of $7.22 \times 10^{17} \mathrm{~cm}^{-2}$, we have a good fit with the observed spectra. We have also estimated the column density of $\mathrm{CH}_{3} \mathrm{NCO}$ using rotation diagram analysis, which is one order higher as compared to LTE model fitting result. $\mathrm{CH}_{3} \mathrm{NCO}$ emissions are also concentrated towards the center of the source's dust continuum. However, all these transitions are marginally resolved. Thus, we can not provide the detailed distributions of this molecule.

\section{DISCUSSIONS}

Synthesis of many COMs (e.g., ethylene glycol, methyl formate, etc.) in G31 has been discussed by Rivilla et al. (2017). They discussed the formation routes of a few COMs. Thus, we do not concentrate on the synthesis of all the species which are identified in the present observation. Here, we mainly focus on the chemistry of two newly reported molecules, $\mathrm{CH}_{3} \mathrm{NCO}$ and $\mathrm{CH}_{3} \mathrm{SH}$ and two other COMs such as $\mathrm{CH}_{3} \mathrm{OH}$ and $\mathrm{CH}_{3} \mathrm{OCHO}$ that 


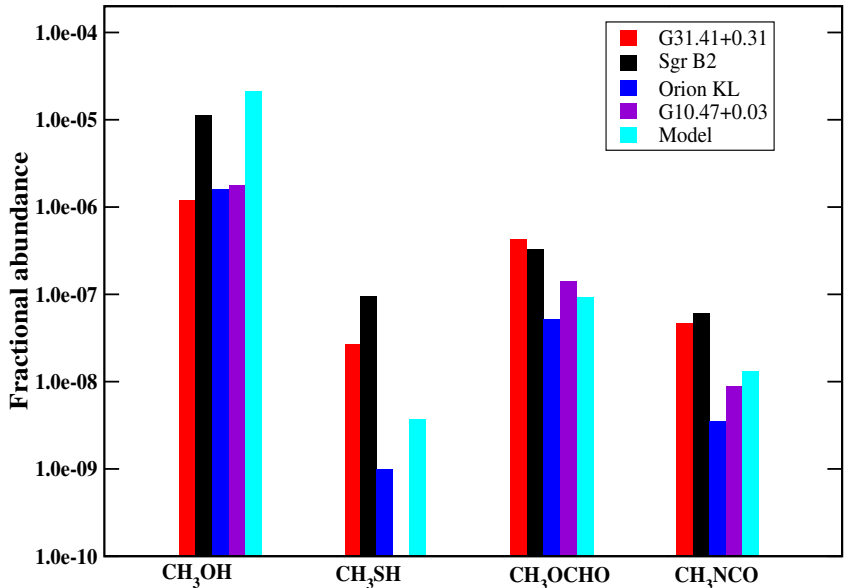

Figure 9. Comparison of abundance of $\mathrm{CH}_{3} \mathrm{OH}, \mathrm{CH}_{3} \mathrm{SH}$, $\mathrm{CH}_{3} \mathrm{CHO}$ and $\mathrm{CH}_{3} \mathrm{NCO}$ between $\mathrm{G} 31$ and other sources (Sgr B2, Orion KL, G10.47+0.03, and model). These abundances are given in the Table 4 with their references.

are discussed in the text. Here, we have discussed the possible synthesis routes of these species and compared their observed abundance/molecular ratio between G31 and other hot molecular cores. We have also shown a comparison between observed results and existing astrochemical simulation of these species. For the modeling results, we mainly followed Garrod et al. (2013). They considered an isothermal collapse phase, which is followed by a static warm-up phase. In the first phase, the density increases from $\mathrm{n}_{\mathrm{H}}=3 \times 10^{3}$ to $10^{7} \mathrm{~cm}^{-3}$ under the free-fall collapse. At the initial phase, dust temperature falls to $8 \mathrm{~K}$ from $16 \mathrm{~K}$. In the second phase; the temperature varies from $8 \mathrm{~K}$ to $400 \mathrm{~K}$, where density remains fixed at $10^{7} \mathrm{~cm}^{-3}$. The temperature of this source is $\sim 150 \mathrm{~K}$, which is typical hot core temperature, and the number density $\left(\mathrm{n}_{\mathrm{H}}\right)$ of this source is $\sim 10^{7} \mathrm{~cm}^{-3}$ (Beltrán et al. 2018). Thus, the hot core model of Garrod et al. (2013) is suitable for understanding chemical evolution of COMs in this source. They used three different warm-up models based on the time scale (i.e., fast, medium, and slow) and the final temperature of their model is much larger than one that is observed in this source. The timescale of the fast warm-up model is more suitable for studying the chemical evolution in high-mass star-forming regions. We used peak gas-phase abundances of all the species around 100-200 K of fast warm-up timescale model to compare with the observed values. Figure 9 compares the observed fractional abundance of $\mathrm{CH}_{3} \mathrm{OH}, \mathrm{CH}_{3} \mathrm{SH}, \mathrm{CH}_{3} \mathrm{OCHO}$, and $\mathrm{CH}_{3} \mathrm{NCO}$ between G31 and other sources. The chemistry of these COMs is discussed in the following subsections.

\section{1. $\mathrm{CH}_{3} \mathrm{OH}$ and $\mathrm{CH}_{3} \mathrm{SH}$}

$\mathrm{CH}_{3} \mathrm{OH}$ and $\mathrm{CH}_{3} \mathrm{SH}$ molecules are the chemical ana$\log$ of each other and have a similar structure only difference is that one has an oxygen atom another sulfur atom. Emitting regions of $\mathrm{CH}_{3} \mathrm{SH}$ transitions are compact and marginally resolved, whereas the emitting region of methanol is slightly extended compared to $\mathrm{CH}_{3} \mathrm{SH}$. A large number of previous experimental, theoretical, and observed results suggest that these two species are mainly produced on the grain surface via successive hydrogen addition reactions (e.g., Das et al. 2008; Fedoseev et al. 2015; Müller et al. 2016; Gorai et al. 2017; Vidal et al. 2017) with CO and CS, respectively. The main route of formation of methanol and methanethiol is given below,

$$
\mathrm{CO} \stackrel{\mathrm{H}}{\rightarrow} \mathrm{HCO} \stackrel{\mathrm{H}}{\rightarrow} \mathrm{H}_{2} \mathrm{CO} \stackrel{\mathrm{H}}{\rightarrow} \mathrm{CH}_{3} \mathrm{O} \stackrel{\mathrm{H}}{\rightarrow} \mathrm{CH}_{3} \mathrm{OH}
$$

and

$$
\mathrm{CS} \stackrel{\mathrm{H}}{\rightarrow} \mathrm{HCS} \stackrel{\mathrm{H}}{\rightarrow} \mathrm{H}_{2} \mathrm{CS} \stackrel{\mathrm{H}}{\rightarrow} \mathrm{CH}_{3} \mathrm{~S} \stackrel{\mathrm{H}}{\rightarrow} \mathrm{CH}_{3} \mathrm{SH} .
$$

For the above reaction schemes, the first and third steps posses an activation barrier, whereas the second and fourth steps are barrier-less (e.g., Hasegawa et al. 1992; Gorai et al. 2017). At low-temperature regions, methanol and methanethiol are efficiently produced on the grain surface and released to the gas phase via nonthermal desorption in the cold phase and during the warm-up stage via thermal desorption process. From the astrochemical simulation, Müller et al. (2016) found that peak abundance of methanol arises around $130 \mathrm{~K}$. Here, our observed results of methanol predict the gas temperature $\sim 180 \mathrm{~K}$, which is similar to the typical hot core temperature. In addition to grain surface reactions, $\mathrm{H}_{2} \mathrm{CS}$ can also be produced in the gas phase. In the gas phase, $\mathrm{H}_{2} \mathrm{CS}$ is mainly produced from $\mathrm{H}_{3} \mathrm{CS}^{+}$, where $\mathrm{H}_{3} \mathrm{CS}^{+}$is formed via the ion-neutral reaction between $\mathrm{S}^{+}$and $\mathrm{CH}_{4}$. Below $\sim 20 \mathrm{~K}$ with a density $\left(\mathrm{n}_{\mathrm{H}}\right)$ around $10^{4}-10^{5} \mathrm{~cm}^{-3}, \mathrm{H}_{2} \mathrm{CS}$ is accreted onto the grain surface directly from gas phase, which may also contribute to the $\mathrm{CH}_{3} \mathrm{SH}$ formation (Bonfand et al. 2019).

The observed abundance of $\mathrm{CH}_{3} \mathrm{SH}$ in $\mathrm{G} 31$ is $2.7 \times 10^{-08}$ and $1.0 \times 10^{-09}$ in Orion KL (Kolesniková et al. 2014, see Table 4). The observed abundance of methanethiol in Sgr B2 is 3.5 times higher than the G31. Methanol abundance is almost similar in G31, Orion KL, G10.47+0.03, and 10 times higher in Sgr B2 (see Figure 9). The observed $\mathrm{CH}_{3} \mathrm{SH} / \mathrm{CH}_{3} \mathrm{OH}$ ratio in G31 is similar to Orion KL and it is eight times higher in Sgr B2. Modeling results of these two species also have comparable abundances and molecular ratio (see Table $4)$. 


\section{2. $\mathrm{CH}_{3} \mathrm{OCHO}$}

Methyl formate can efficiently be produced on the surface of dust-grains through the reaction between methoxy radical $\left(\mathrm{CH}_{3} \mathrm{O}\right)$ and formyl radical (HCO) (Garrod et al. 2008, 2013). Their simulation shows that, around $30-40 \mathrm{~K}$, these radicals are mobile, and the reaction is efficient. It is clear from their simulation that gas phase $\mathrm{CH}_{3} \mathrm{OCHO}$ is mainly coming from the ice phase (see Figure 1 of Garrod et al. 2013). Increased UV photodissociation of $\mathrm{CH}_{3} \mathrm{OH}$ leads to the formation of various radicals $\mathrm{CH}_{3}, \mathrm{CH}_{3} \mathrm{O}$, and $\mathrm{CH}_{2} \mathrm{O}$, around $40 \mathrm{~K}$ temperature, these are mobile and form the COMs (e.g., $\mathrm{CH}_{3} \mathrm{OCHO}, \mathrm{CH}_{3} \mathrm{OCH}_{3}$ ). In the gas phase, when $\mathrm{T} \sim 40 \mathrm{~K}$, protonated methanol and $\mathrm{H}_{2} \mathrm{CO}$ (when it is abundantly released to the gas phase) react and form $\mathrm{HC}(\mathrm{OH}) \mathrm{OCH}_{3}{ }^{+}$. Then electron recombination of $\mathrm{HC}(\mathrm{OH}) \mathrm{OCH}_{3}+$ produce $\mathrm{CH}_{3} \mathrm{OCHO}$ (Bonfand et al. 2019). An efficient gas-phase reaction of methyl formate production was proposed by Balucani et al. (2015) for the cold environment, where dimethyl ether is the precursor of methyl formate. Dimethyl ether is also present in this source (Isokoski, Bottinelli \& van Dishoeck. 2013). Therefore methyl formate can be produced through both grain surface and gas-phase reactions. The observed abundance of $\mathrm{CH}_{3} \mathrm{OCHO}$ in G31 is $4.25 \times 10^{-7}$, which is similar to the observed abundance in Sgr B2, G10.47+0.03 and ten times higher as compared to the Orion $\mathrm{KL}$ and modeling results. In G31, we have observed $\mathrm{CH}_{3} \mathrm{OCHO} / \mathrm{CH}_{3} \mathrm{OH}$ abundance ratio $\sim 0.14-0.35$. For other high-mass star-forming regions, this ratio is found to be 0.03, 0.03, and 0.08 in Sgr B2, Orion KL, and G10.47+0.03, respectively (see Table 4).

\section{3. $\mathrm{CH}_{3} \mathrm{NCO}$}

In this work, we find emitting regions of $\mathrm{CH}_{3} \mathrm{NCO}$ is compact. In this source, Rivilla et al. (2017) also observed a similar emitting region of ethylene glycol. It is primarily produced on the grain surface via recombination of two HCO radicals followed by successive hydrogen addition reactions (Coutens et al. 2017). In case of $\mathrm{CH}_{3} \mathrm{NCO}$ formation, Halfen et al. (2015) proposed two routes (i) reaction between $\mathrm{HNCO}(\mathrm{HOCN})$ and $\mathrm{CH}_{3}$, and (ii) reaction between $\mathrm{HNCO}(\mathrm{HOCN})$ and $\mathrm{CH}_{5}^{+}$. Cernicharo et al. (2016) proposed that it could be produced on the grain surface. The experiment result suggests that $\mathrm{CH}_{3} \mathrm{NCO}$ can efficiently be produced on the solid phase through the reaction between $\mathrm{CH}_{3}$ and (H)NCO (Ligterink et al. 2017). The hot core model suggests that it could be produced via a radical-radical reaction between $\mathrm{CH}_{3}$ and OCN (Belloche et al. 2017). Since the reaction mentioned above is exothermic and barrier-less, it can efficiently be produced on the grain surface at a little warmer temperature $(>30 \mathrm{~K})$, when radical get enough mobility. Though $\mathrm{CH}_{3} \mathrm{NCO}$ is efficiently produced on the grain surface, it could also be destructed by hydrogen addition reactions and produce n-methyl formamide (Belloche et al. 2017). Quénard et al. (2018) also considered similar radical-radical reactions in their chemical model to study the evolution of $\mathrm{CH}_{3} \mathrm{NCO}$ under hot core conditions. In the hot core, when the temperature reaches around $100 \mathrm{~K}, \mathrm{CH}_{3}$ and HNCO can thermally desorb from the grain surface and increase the formation of $\mathrm{CH}_{3} \mathrm{NCO}$ (MartínDoménech et al. 2017; Gorai et al. 2020). Our estimated $\mathrm{CH}_{3} \mathrm{NCO}$ abundance in G31 is $\sim 4.72 \times 10^{-8}$. For other hot molecular cores (e.g., Sgr B2, Orion KL), we have found a comparable abundance of $\mathrm{CH}_{3} \mathrm{NCO}$. The observed fractional abundance of $\mathrm{CH}_{3} \mathrm{NCO}$ in this work is well within the modeled results (model 5) of Belloche et al. (2017).

The identification of $\mathrm{CH}_{3} \mathrm{OH}, \mathrm{CH}_{3} \mathrm{OCHO}, \mathrm{CH}_{3} \mathrm{NCO}$, and $\mathrm{CH}_{3} \mathrm{SH}$ in $\mathrm{G} 31$ suggesting both grain surface and gas phase chemistry is efficient in this source for the formation of COMs. However, we can not distinguish the contribution from the gas and grain phase separately due to the observational limit. To explain the various observed line profile in this work, it requires a combined chemical and radiative transfer model, which will be carried out in our follow-up study.

\section{CONCLUSIONS}

We analyzed ALMA band 3 data of a hot molecular core, G31.41+0.31, and the following conclusions are made.

- We detected methyl isocyanate $\left(\mathrm{CH}_{3} \mathrm{NCO}\right)$ and methanethiol $\left(\mathrm{CH}_{3} \mathrm{SH}\right)$, for the first time in G31.

- We obtained two temperature components by using rotation diagram analysis. The cold component is obtained by $\mathrm{CH}_{3} \mathrm{OH}$ and $\mathrm{CH}_{3} \mathrm{NCO}$ where temperature varies between 33 and $48 \mathrm{~K}$. Hot component is obtained by $\mathrm{CH}_{3} \mathrm{SH}, \mathrm{CH}_{3} \mathrm{OH}$ and $\mathrm{CH}_{3} \mathrm{OCHO}$ where the temperature ranges between 143 and $181 \mathrm{~K}$. Estimated hydrogen $\left(\mathrm{N}_{\mathrm{H}_{2}}\right)$ column density is $\sim 1.53 \times 10^{25} \mathrm{~cm}^{-2}$ and dust is optically thin at $94 \mathrm{GHz}$.

- We estimated column density and fractional abundances of various complex species $\mathrm{CH}_{3} \mathrm{NCO}$, $\mathrm{CH}_{3} \mathrm{SH}, \mathrm{CH}_{3} \mathrm{OH}$, and $\mathrm{CH}_{3} \mathrm{OCHO}$ in G31. A comparison of column density/fractional abundances of these species between G31 and other hot molecular cores is discussed. We also compared our 
observed results with available chemical modeling under hot core conditions. Our observed abundances of COMs in G31 are consistent with other hot cores, Sgr B2, Orion KL, and G10.47+0.03, which suggests similar formation mechanisms of these complex species in these sources.

- For the kinematics of this source, a low excitation line of $\mathrm{H}^{13} \mathrm{CO}^{+}$is observed as an inverse P-Cygni profile, which suggests that there is an infall toward the center of this source, i.e., this source is in the younger stage of its evolution.

- Protostellar outflows are traced by SiO and HCN. Their blue-shifted and red-shifted emission clearly trace outflow along East-West direction. We estimated various outflow parameters. The average dynamical time scale of outflow is $\sim 10^{3}$ years.

\section{ACKNOWLEDGEMENT}

This paper makes use of the following ALMA data: ADS/JAO.ALMA\#2015.1.01193.S. ALMA is a partnership of ESO (representing its member states), NSF (USA), and NINS (Japan), together with NRC
(Canada), NSC, and ASIAA (Taiwan), and KASI (Republic of Korea), in cooperation with the Republic of Chile. The Joint ALMA Observatory is operated by ESO, AUI/NRAO, and NAOJ. We are grateful to the anonymous referee for numerous comments and suggestions, which improved the manuscript. PG acknowledges CSIR extended SRF fellowship (Grant No. 09/904 (0013) 2018 EMR-I) and and Chalmers Cosmic Origins postdoctoral fellowship. AD acknowledges ISRO respond project (Grant No. ISRO/RES/2/402/16-17) for partial financial support. B.B. acknowledges DSTINSPIRE Fellowship [IF170046] for providing partial financial assistance. SKM acknowledges CSIR fellowship (Ref no. 18/06/2017(i) EU-V). This work is partially supported by Consortium for the Development of Human Resources in Science and Technology by Japan Science and Technology Agency. This research was possible in part due to a Grant-In-Aid from the Higher Education Department of the Government of West Bengal. DS acknowledges ASIAA postdoctoral research fund and travel grant. AD and TS also acknowledge support from DST JSPS grant.

Software: CASA (v4.7.2; McMullin et al. 2007), CASSIS (Cassis Team At CESR/IRAP 2014), RADEX (Van der Tak et al. 2007)

\section{REFERENCES}

Araya, E., Hofner, P., Kurtz, P., Olmi, L. \& Linz, H, 2008, ApJ, 675, 420

Altwegg K., Balsiger, H., Berthelieret, J. J. et al. 2017, MNRAS, 469, S130

Estalella, R., Anglada, G., Díaz-Rodríguez, A. K., Mayen-Gijon, J. M. 2019, A\&A, 626, A84

Balucani N., Ceccarelli C., Taquet V. 2015, MNRAS, 449, L16

Belloche, A., Menten, K. M., Comito, C. et al. 2008, A\&A, 482, 179

Belloche, A., Müller, H. S. P., Menten, K. M. et al. 2013, A\&A, 559, A47

Belloche, A., Garrod, R.T. , Müller, H. S. P., \& Menten, K. M. 2014, Science, 345, 158

Belloche, A., Müller, H. S. P., Garrod, R. T., \& Menten, K. M. 2016, A\&A, 587, A91

Belloche, A., Meshcheryakov, A. A., Garrodet, R. T. et al. 2017, A\&A, 601, A49

Beltrán M. T., Cesaroni R., Neri R. et al. 2005, A\&A, 435, 90

Beltrán, M.T., Codella, C., S. Viti, S. et al. 2009, 690, L93

Beltrán, M. T. \& de Wit, W. J. 2016, A\&A Rev., 24, 6

Beltrán, M. T., Cesaroni, R., Rivilla, V. M. et al. 2018, A\&A, 615,141

Beltrán et al. 2019, A\&A, 630, 54

Blake, G. A., Sutton, E., Masson, C., \& Phillips, T. 1987, ApJ, 315, 621

Boehler, Y., Weaver, E., Isella, A., et al. 2017, ApJ, 840, 60

Bonfand, M., Belloche, A., Menten, K. M. et al. 2017, A\&A, 604, 60

Bonfand, M., Belloche, A., Garrod, R.T., et al. A\&A, 628, A27

Brown, R.D., Crofts, J.G., Godfrey, P.D. et al. 1975, ApJ, 197, L29.

Cabrit, S., \& Bertout, C. 1992, A\&A, 261, 276

Calmonte, U., Altwegg, K., Balsiger, H. et al. 2016, MNRAS, 462, S253

Casassus, S., van der Plas, G., M, S. P., et al. 2013, Nature, 493, 191

Cazaux, S., Tielens, A. G. G. M., Ceccarelli, C. et al. 2003, ApJ. 593, L51

Cernicharo J., Kisiel, Z., Tercero, B., et al., 2016, A\&A, 587, L4

Cesaroni, R., Hofner, P., Araya, E., \& Kurtz, S. 2010, A\&A, 509, 50
Cesaroni, R., Beltrán, M. T., Zhang, Q. et al. 2011, A\&A, 533, A73 Cesaroni, R., Sánchez-Monge, Á., Beltrán, M. T., et al. 2017, A\&A, 602, A59

Charnley, S. B. 1997, ApJ, 481, 396

Chakrabarti, S.K., Majumdar, L., Das, A., Chakrabarti, S., 2015, APSS, 2015, 357, 90

Churchwell E., Walmsley C. M., Cesaroni R., 1990, A\&AS, 83, 119

Coutens, A., Viti, S., Rawlings, J.M.C. et al. 2017, MNRAS, 475, 2016

Codella, C., Beltrán, M. T., Cesaroni, R., et al. 2013, A\&A, 550, A81

Cox, A. N. 2000, Allen's Astrophysical Quantities (New York: AIP Press)

Daly, A., Bermúdez, C., López, A. et al. 2013, ApJ, 768, 81

Das, A., Acharyya, K., Chakrabarti, S. \& Chakrabarti, S. K. 2008b, A\&A, 486, 209

Das, A., Acharyya, K. \& Chakrabarti, S. K., 2010, MNRAS 409, 789

Das, A. \& Chakrabarti, S. K., 2011, MNRAS, 418, 545

Das, A., Sahu, D., Majumdar, L., \& Chakrabarti, S. K., 2016, MNRAS, 455, 540.

Demyk, K., Mäder, H.; Tercero, B. et al. 2007, A\&A, 466, 255

Di Francesco, J., Myers, P. C., Wilner, D. J. et al. 2001, ApJ, 562, 770

Esplugues, G. B., Tercero, B., Cernicharo, J., et al. 2013, A\&A, 556, A 143

Favre, C., Despois, D., Brouillet, N., et al. 2011, A\&A, 532, A32

Favre, C., Bergin, E. A., Neill, J. L., et al. 2015, ApJ, 808, 155

Fedoseev, G., Cuppen, H. M., Ioppolo, S., Lamberts, T., \& Linnartz, H. 2015, MNRAS, 448, 1288

Garrod R. T., Weaver S. L. W., Herbst E., 2008, ApJ, 682, 283

Garrod R. T., 2013, ApJS, 765, 60

Gibb, E., Nummelin, A., Irvine, W. M. et al. 2000, ApJ, 545, 309

Girart, J. M., Beltrán, M. T., Zhang, Q. et al. 2009, Science, 324, 1408

Girart, J. M., Fernández-López, M., Li, Z. -Y., et al. 2018, ApJL, $856, \mathrm{~L} 27$ 
Goesmann F. et al., 2015, Science, 346, 6247

Gorai, P., Das, A., Das, A., et al. 2017, ApJ, 836, 70

Gorai, P., Bhat, B., Sil, M., Mondal, S. K., Ghosh, R., Das, A., 2020 , ApJ, 895, 86

Goldsmith, P. F., \& Langer, W. D. 1999, ApJ, 517, 209

Halfen D. T., Ilyushin V. V., Ziurys L. M., 2015, ApJ, 812, L5

Hasegawa, T. I., Herbst, E. \& Leung, C. M. 1992, ApJS, 82, 167

Herbst, E. \& van Dishoeck, E. F. 2009, ARA\&A, 47, 427

Higuchi, A. E., Hasegawa, T., Saigo, K. et al. J. O. 2015, ApJ, 815, 106

Hollis, J, M., Jewell., P.R., Lovas, F.J.,\& Remijan, A. 2004, ApJ, 613, L45

Isella, A., Guidi, G., Testi, L., et al. 2016, PhRvL, 117, 25

Isokoski K., Bottinelli S., van Dishoeck E. F., 2013, A\&A, 554, A100

Jin, S., Li, S., Isella, A., Li, H., \& Ji, J. 2016, ApJ, 818, 76

Kolesniková, L., Tercero, B., Cernicharo, J., et al. 2014, ApJ, 784, L7

Kraus, S., Hofmann, K-H., Menten, K. M., et al. 2010, Nature, 466, 339

Kurtz S., Cesaroni R., Churchwell E. et al. 2000, Protostars and Planets IV ed V. Mannings, A. P. Boss and S. S. Russell (Tucson, AZ: Univ. Arizona Press) 299

Leurini, S., Beuther, H., Schilke, P. et al. 2007, A\&A, 475, 925

Leurini, S., Codella, C., López-Sepulcre, A. et al. 2014, A\&A, 570 , A49

Ligterink, N. F. W, Tenenbaum, E. D., \& van Dishoeck, E. F, 2015, A\&A, 576, A35

Ligterink N. F. W., et al., 2017, MNRAS, 469, 2219

Linke, R. A., Frerking, M. A., \& Thaddeus, P., 1979, ApJ, 234, L139

Majumdar, L., Gorai, P., Das, Ankan., Chakrabarti, S. K., 2015 APSS, 360, 64

McGuire, B. A., Carroll, P. B., Loomis, R. A. et al. 2016, Science, 352, 1449-1552.

McMullin, J. P., Waters, B., Schiebel, D., Young, W., \& Golap, K. 2007, Astronomical Data Analysis Software and Systems XVI (ASP Conf. Ser. 376), ed. R. A. Shaw, F. Hill, \& D. J. Bell (San Francisco, CA: ASP), 127

Margulés, L, Belloche, A., Müller, H.S.P., Motiyenko, R. A. et al. A\&A, 2016

Martín-Doménech, R., Rivilla, V. M., Jiménez-Serra, I., et al. 2017, MNRAS, 469, 2230

Müller, H.S.P., Belloche, A., Xu, L. et al. 2016, A\&A, 587, A92

Müller, H. S. P., Belloche, A., Menten, K. M., et al. 2008, J. Mol. Spectr., 251, 319
Müller, H. S. P., Thorwirth, S., Roth, D. A., \& Winnewisser, G. 2001, A\&A 370, L49

Müller, H. S. P., Schlöder, F., Stutzki, J., \& Winnewisser, G. 2005 , J. Mol. Str, 742, 215

Mayen-Gijon, J. M. Anglada, G., Osorio, M., et al. 2014, MNRAS, 437,3766

Öberg K. I., Boogert A. C. A., Pontoppidan K. M., et al. eds, IAU Symposium Vol. 280, 2011, The Molecular Universe. pp 65(arXiv:1107.5826), doi:10.1017/S1743921311024872

Ossenkopf, V., \& Henning, T. 1994, A\&A, 291, 943

Olmi, L., Cesaroni, R., \& Walmsley, C. M. 1996, A\&A, 307, 599

Oya, Y., Nami Sakai, N., López-Sepulcre, A. et al. 2016, ApJ, 824, 88

Osorio, M., Anglada, G., Lizano, S. et al. 2009, ApJ, 694, 29.

Patel, N. A., Curiel, S., Sridharan, T. K et al. 2005, Nature, 437, 109

Pickett, H. M., Poynter, R. L., Cohen, E. A. et al. 1998, JQSRT, 60, 883

Pirogov, L., Zinchencko, I., Lapinov, A. et al. 1995, A\&AS, 09, 333

Quénard, D., Jiménez-Serra, I., Viti, S.,et al. 2018, MNRAS, 474 2796

Reid, M., et al. 2019, ApJ, 885, 131

Rivilla, V.M., Beltrán, M.T., Cesaroni, R. et al. 2017, A\&A, 598, 59

Rolffs, R., Schilke, P., Zhang, Q., \& Zapata, L., 2011, A\&A, 536, A33

Schilke, P., Benford, D. J., Hunter, T. R. et al. 2001, ApJS, 132, 281

Sahu, D., Minh., Y.C., Lee, C-F. et al. 2018, MNRAS, 475, 5322

Sahu, D., Liu, S-Y., Das, A., et al. 2020, ApJ, 899, 65

Sil, M., Gorai, P., Das, A. et al. ApJ, 2018, 853,

Snyder, L. E., \& Buhl, D. 1971, ApJ. 163, L47

Sutton, E. C., Peng, R., Danchi, W.C. et al. 1995, ApJS, 97, 455

Tan, J. C., Beltrán, M. T., Caselli, P. et al. 2014, Protostars and Planets VI, 149

Tercero, B., Cuadrado, S., López, A., et al. 2018, A\&A, 620, L6

Vidal, T. H. G., Loison, J-C., Jaziri, A. Y., et al. 2017, MNRAS, 469,435

van Dishoeck E. F., Blake G. A. 1998, Annu Rev Astron Astrophys, 36,317

Van der Tak, F. F. S., Black, J. H., Schöier, et al. 2007, A\&A 468, 627

van der Marel, N., van Dishoeck, E. F., Bruderer, S., et al. 2016, A\&A 585, A58

Weaver, E., Isella, A., \& Boehler, Y. 2018, ApJ, 853, 113

Whittet, D. C. B. 1992, Dust in the Galactic Environment (Bristol: IOP Publishing)

Ziurys, L. M., \& Turner, B. E. 1986, ApJ, 300, L19

Zapata, L. A., Ho, P. T. P., Schilke, P. et al. 2009, ApJ, 698, 1422 


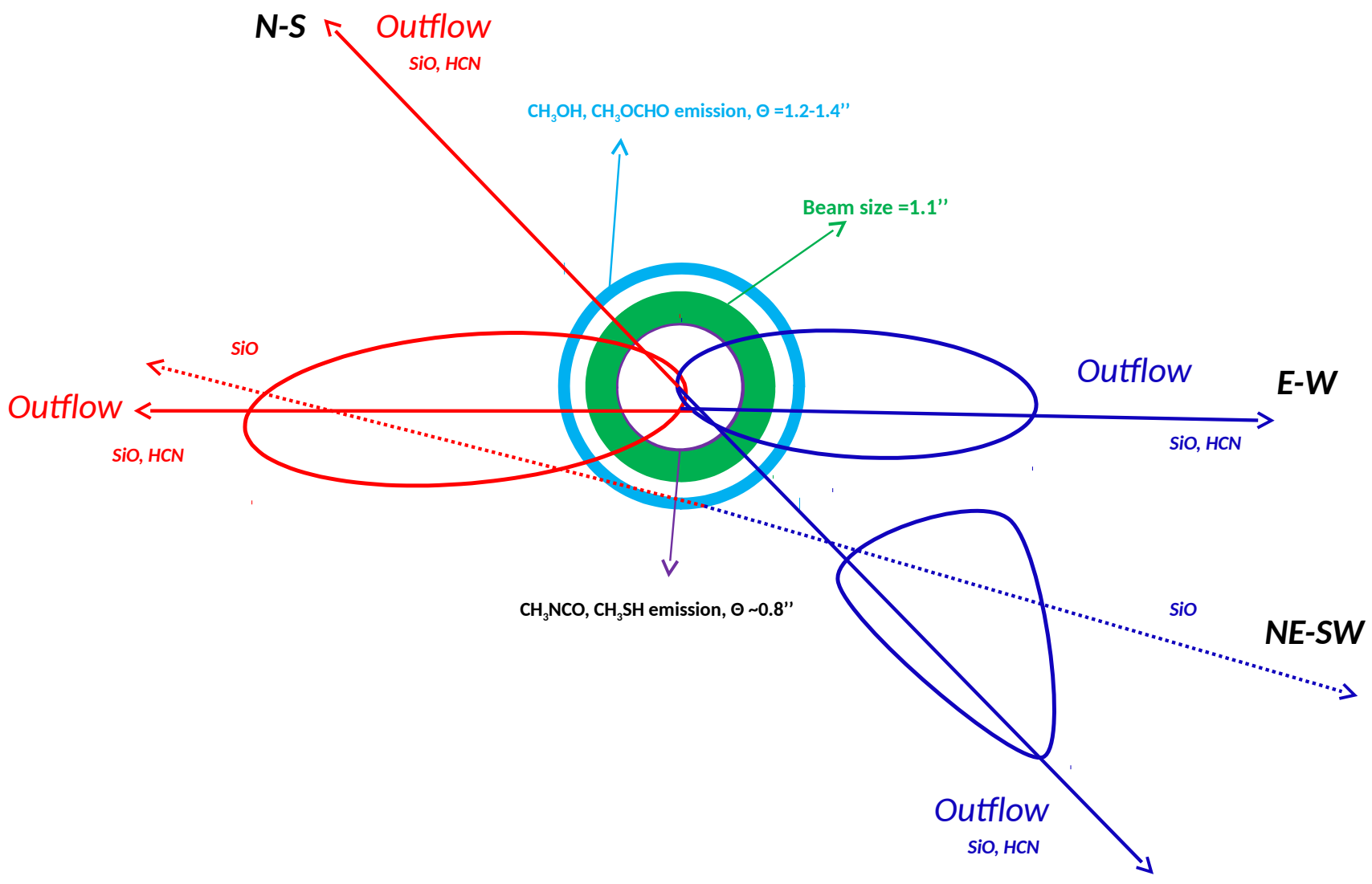

Figure A1. Schematic diagram (not up to scale) of kinematics and molecular distribution in G31.41+0.31 based on present observational results (see details in the Section 3.6.6).

\section{APPENDIX}

A. FITTED SPECTRA, LTE MODEL SPECTRA, MOMENT MAPS, AND SCHEMATIC DIAGRAM OF G31 BASED ON PRESENT OBSERVATION. 
Frequency [GHz]

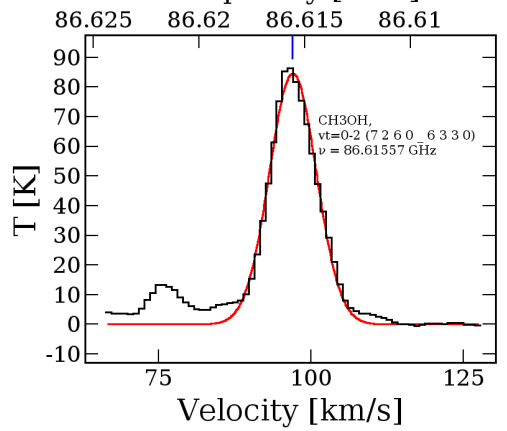

Frequency [GHz]
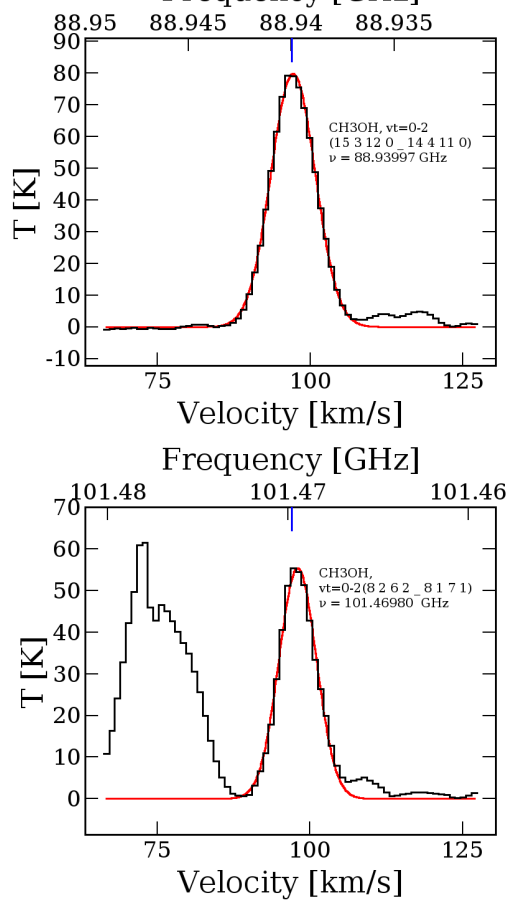

Frequency [GHz]

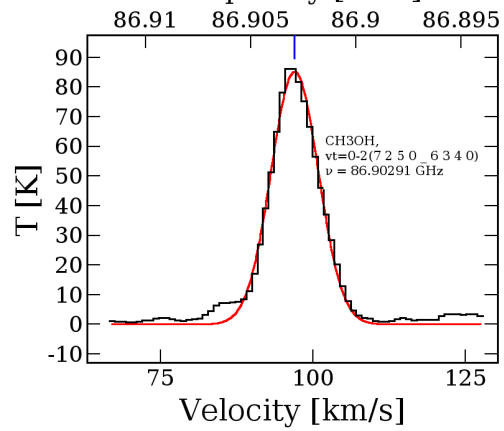

Frequency [GHz]

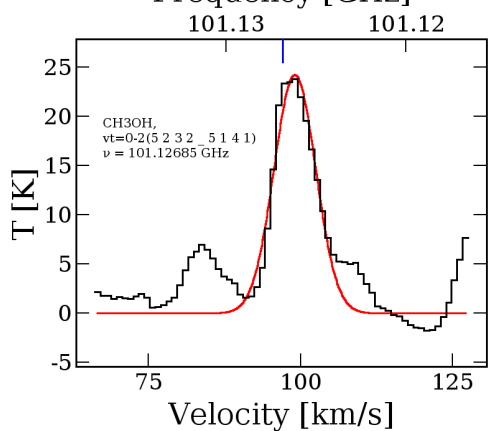

Frequency [GHz]

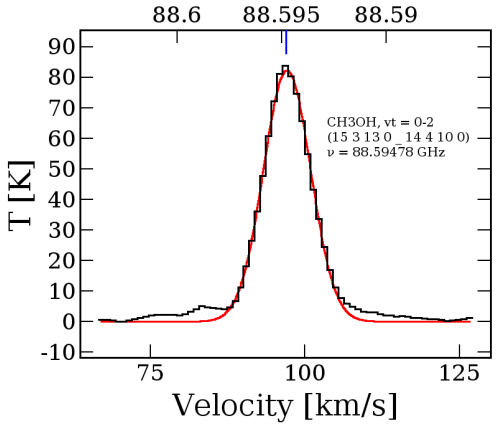

Frequency [GHz]

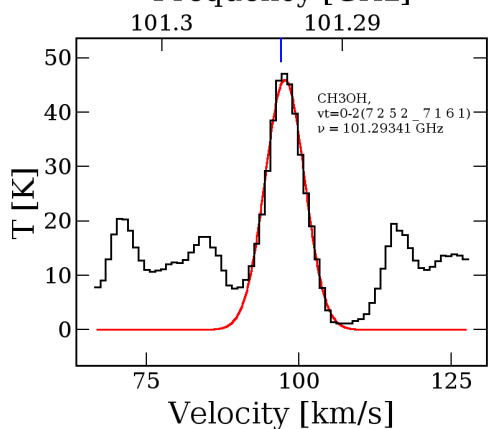

Figure A2. Black line represents observed emission spectra of methanol $\left(\mathrm{CH}_{3} \mathrm{OH}\right)$ and red line represents a Gaussian profile fitted to the observed spectra. 
Frequency [GHz]

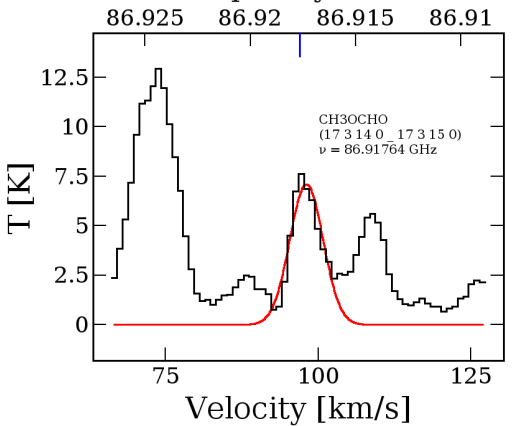

Frequency [GHz]

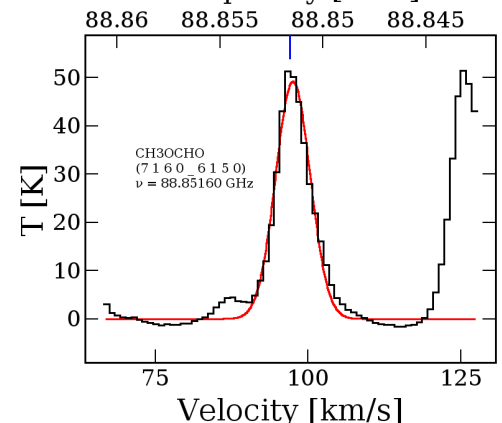

Frequency [GHz]

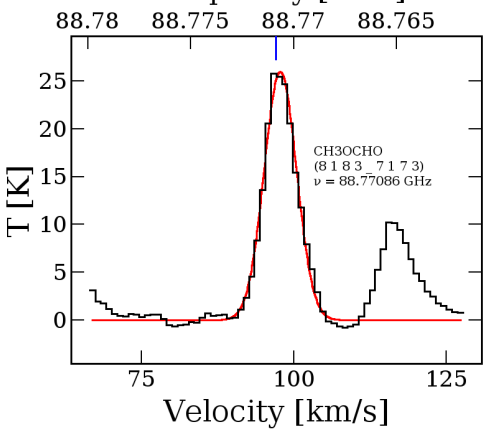

Frequency [GHz]

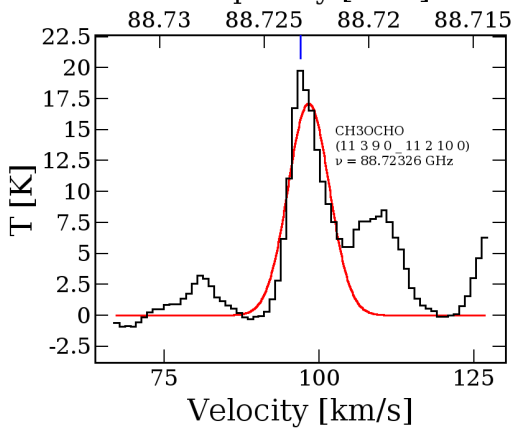

Frequency [MHz]

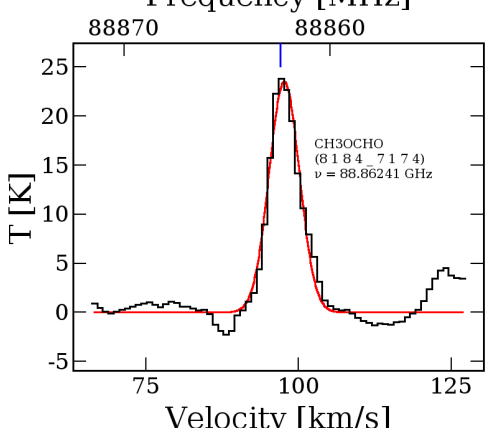

Frequency [GHz]

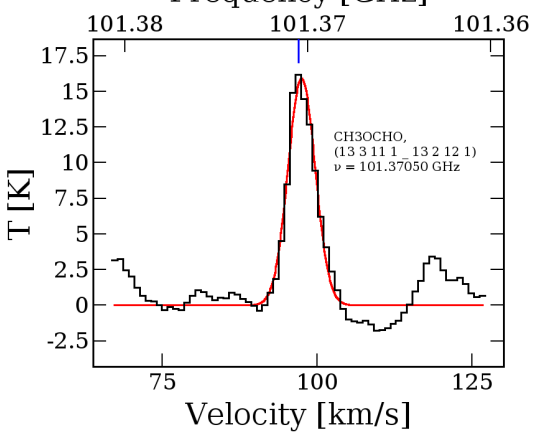

Frequency [GHz]

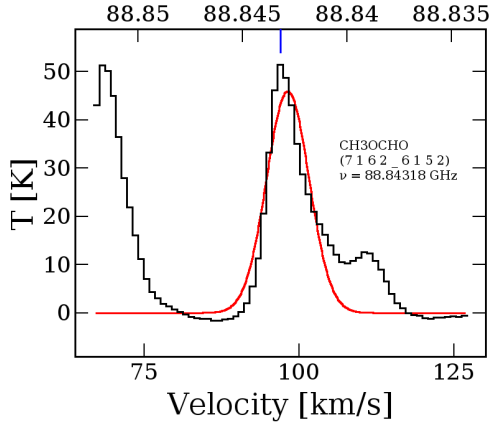

Frequency [GHz]

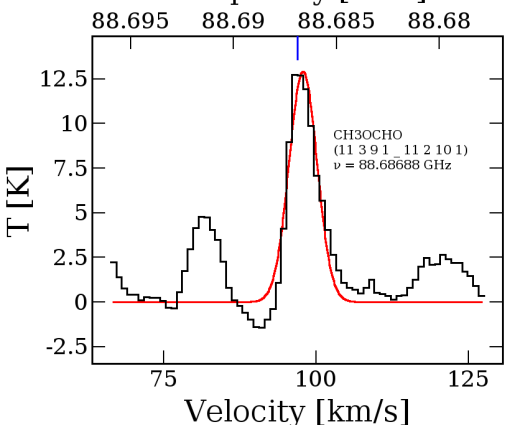

Frequency [GHz]

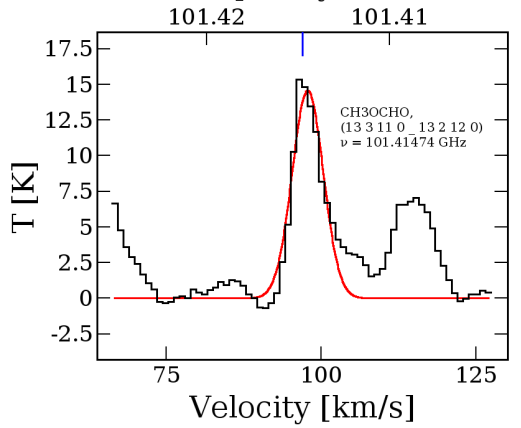

Figure A3. Black line represents observed emission spectra of methyl formate $\left(\mathrm{CH}_{3} \mathrm{OCHO}\right)$ and red line represents a Gaussian profile fitted to the observed spectra. $11_{39}-11_{2,10}(\mathrm{~A})$ transition may blend with some other molecular transition. 

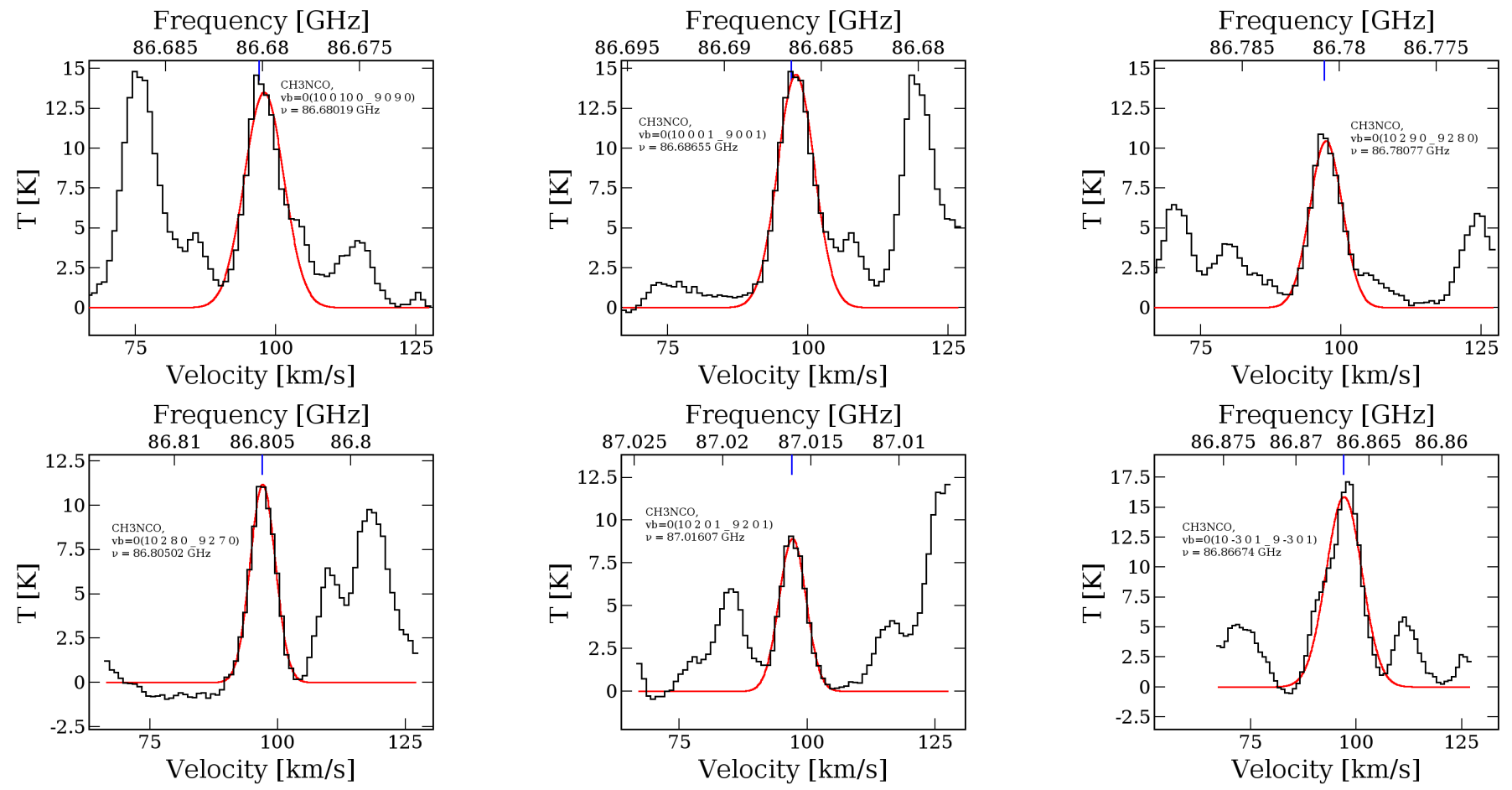

Figure A4. Black line represents observed emission spectra of methyl isocyanate $\left(\mathrm{CH}_{3} \mathrm{NCO}\right)$ and red line represents a Gaussian profile fitted to the observed spectra. $10_{-30}-9_{-30}$ transition may strongly blend with some other molecular transitions.
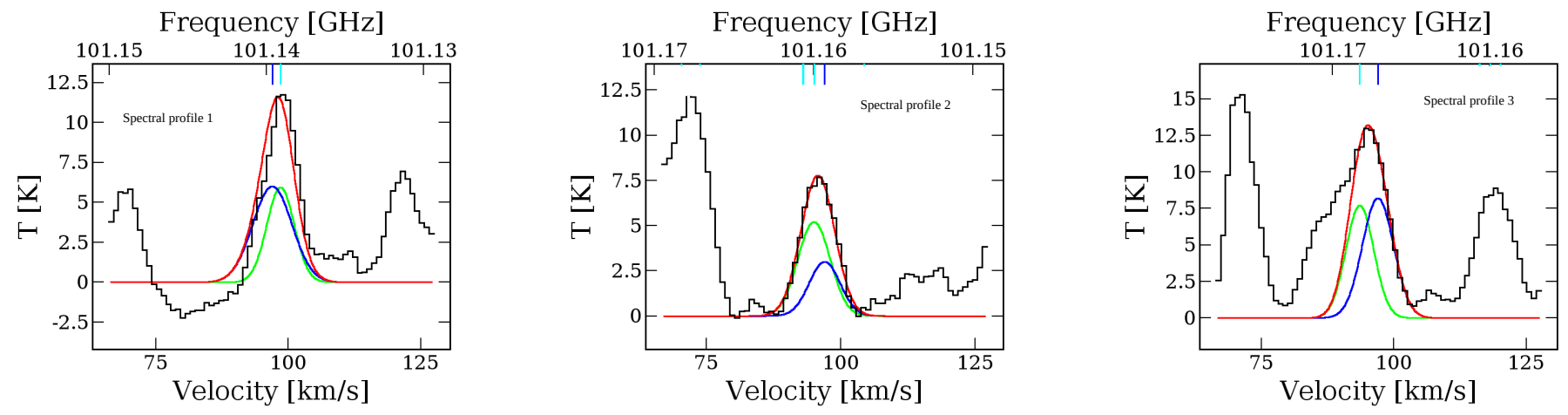

Figure A5. Black line represents observed emission spectra of methanethiol $\left(\mathrm{CH}_{3} \mathrm{SH}\right)$ and red line represents the resultant Gaussian profile of multiple Gaussian components fitted to the observed spectra. In the left panel, blue profile represent the $101.13915 \mathrm{GHz}$ line and green line represent the $101.13965 \mathrm{GHz}$ transition. In the middle panel, though we observe four transitions, we consider two Gaussian components one (blue profile) for $101.15933 \mathrm{GHz}$ and another one (green profile) for other three multiplets $(101.15999 \mathrm{GHz}, 101.16066 \mathrm{GHz}$, and $101.16069 \mathrm{GHz})$. In the right panel, blue and green lines represent 101.16715 GHz and 101.16830 GHz transitions respectively. 

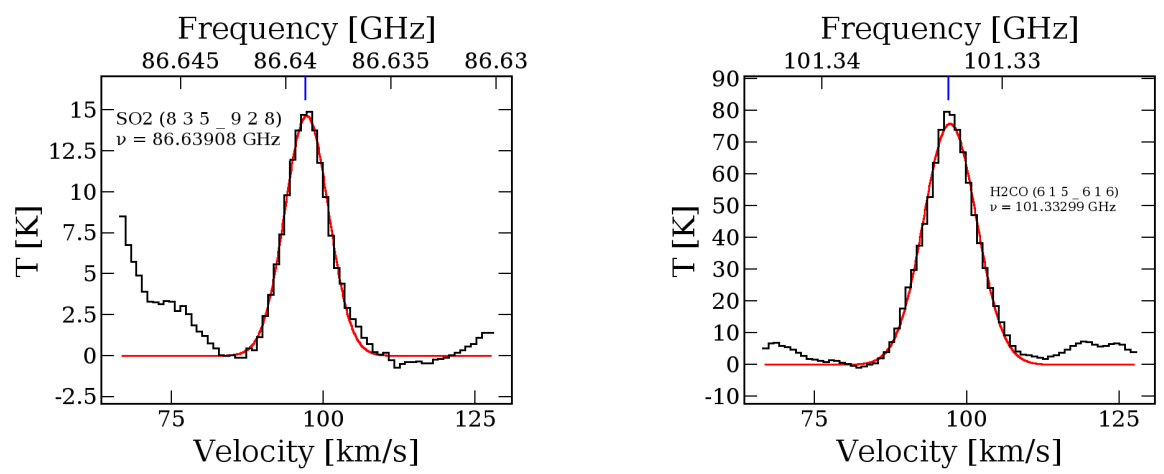

Figure A6. Black line represents observed emission spectra of $\mathrm{SO}_{2}$, and $\mathrm{H}_{2} \mathrm{CO}$ and red line represents a Gaussian profile fitted to the observed spectra.

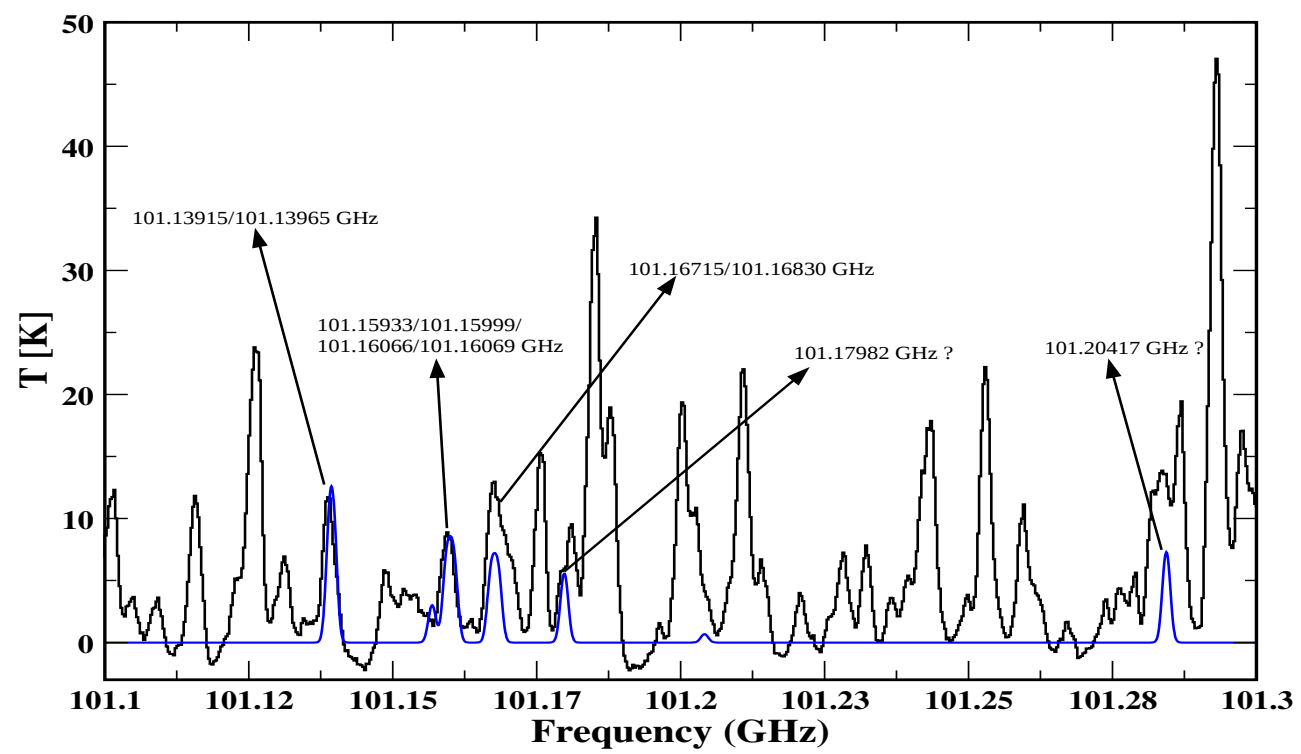

Figure A7. Black line represents observed spectra and blue line represents the (LTE) model spectra of methanethiol $\left(\mathrm{CH}_{3} \mathrm{SH}\right)$. In addition to the observed transitions of $\mathrm{CH}_{3} \mathrm{SH}$ listed in Table 1, we have also see signature of two other transitions (101.17982 $\mathrm{GHz}$ and $101.20417 \mathrm{GHz}$ ), which are heavily blended with other species. 

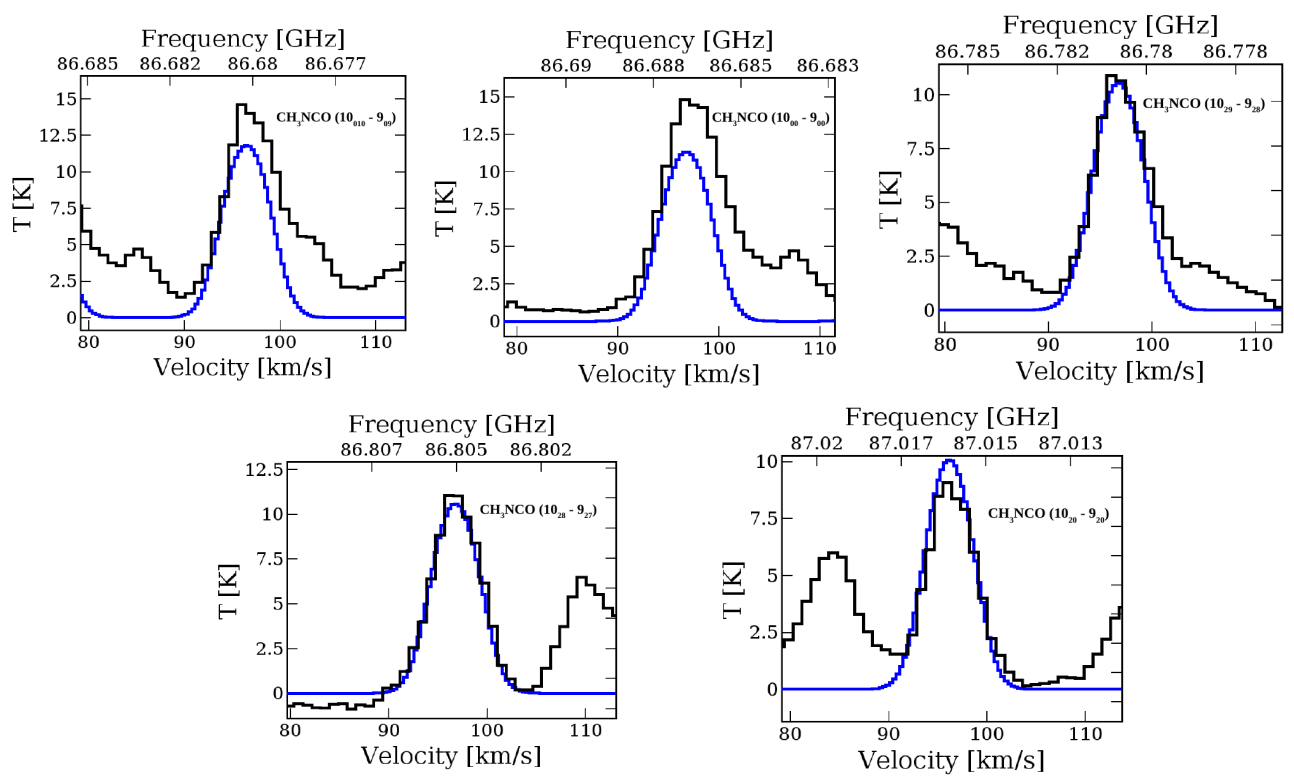

Figure A8. Black line represents observed spectra and blue line represents the (LTE) model spectra of methyl isocyanate $\left(\mathrm{CH}_{3} \mathrm{NCO}\right)$. 

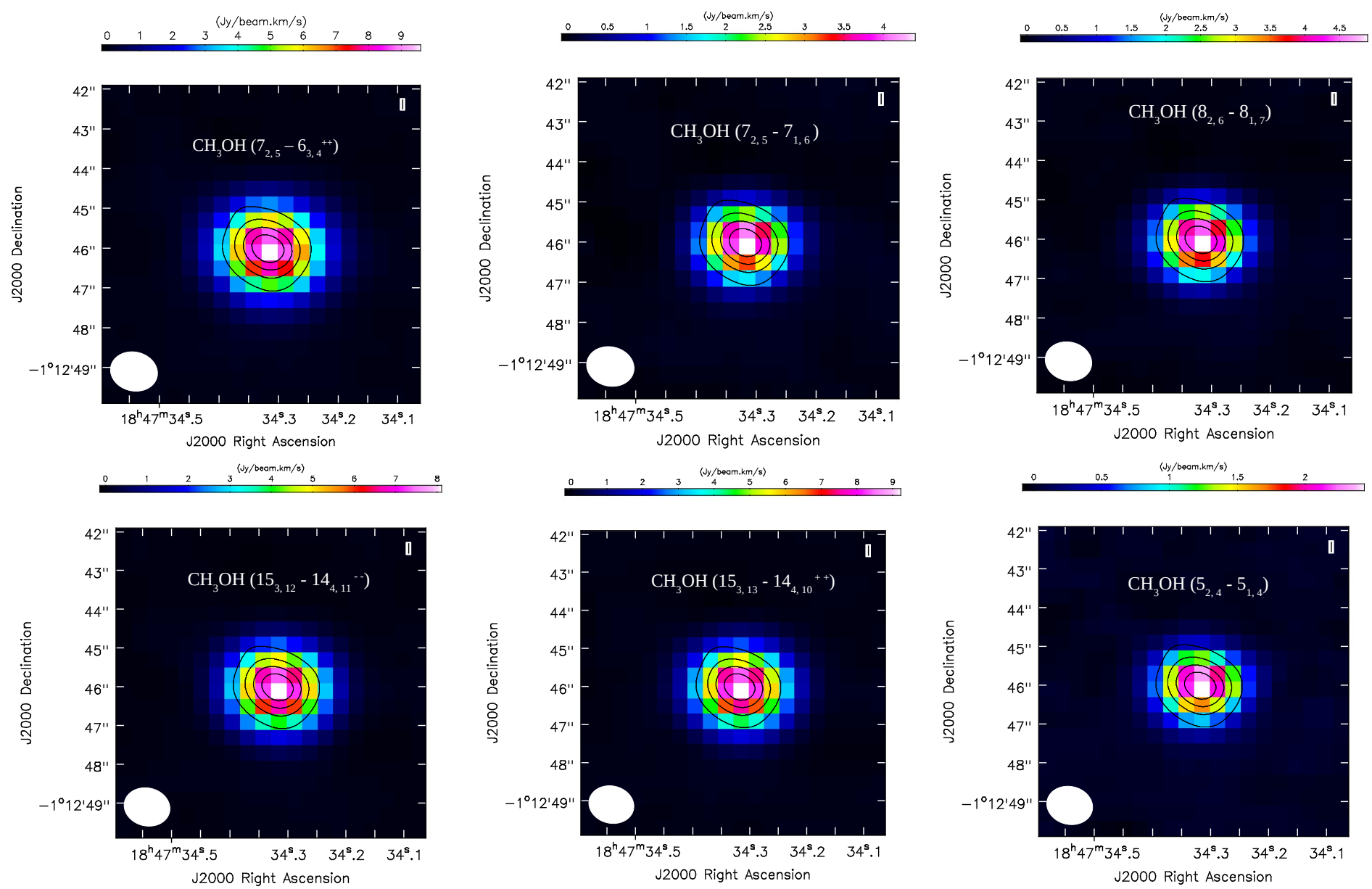

Figure A9. Moment 0 maps of the integrated intensity distribution (color) of $\mathrm{CH}_{3} \mathrm{OH}$ transitions is overlaid on the 3.1 mm continuum emission (black contours). Contour levels are at 20\%,40\%,60\%, and $80 \%$ of the peak flux of the continuum image. The synthesized beam is shown in the lower left-hand corner of each figure. 

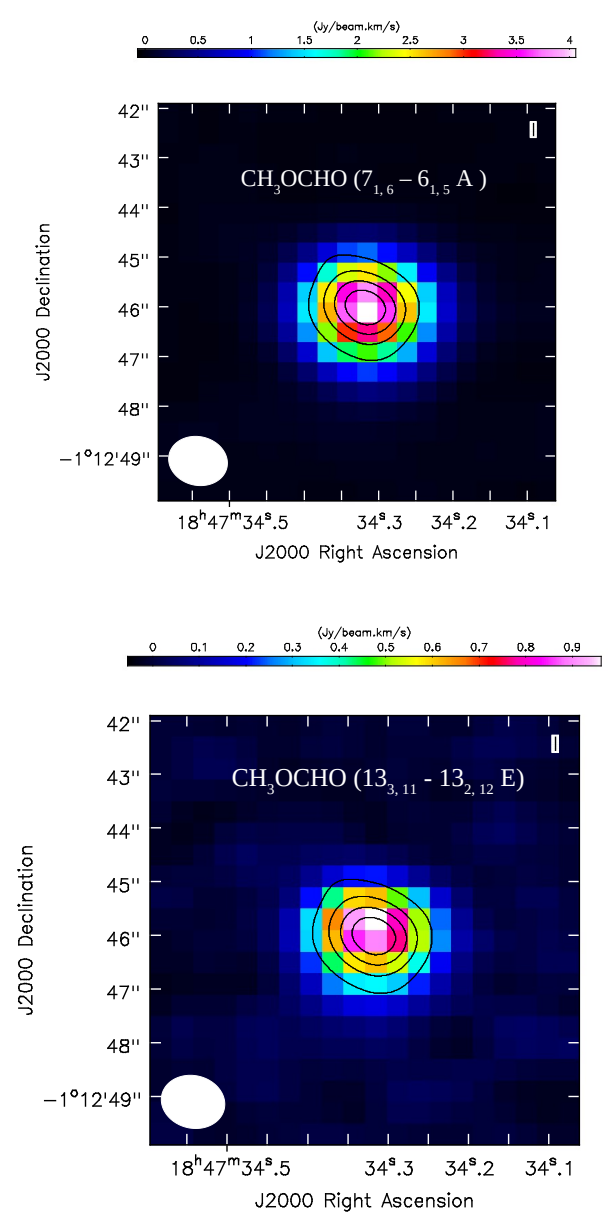
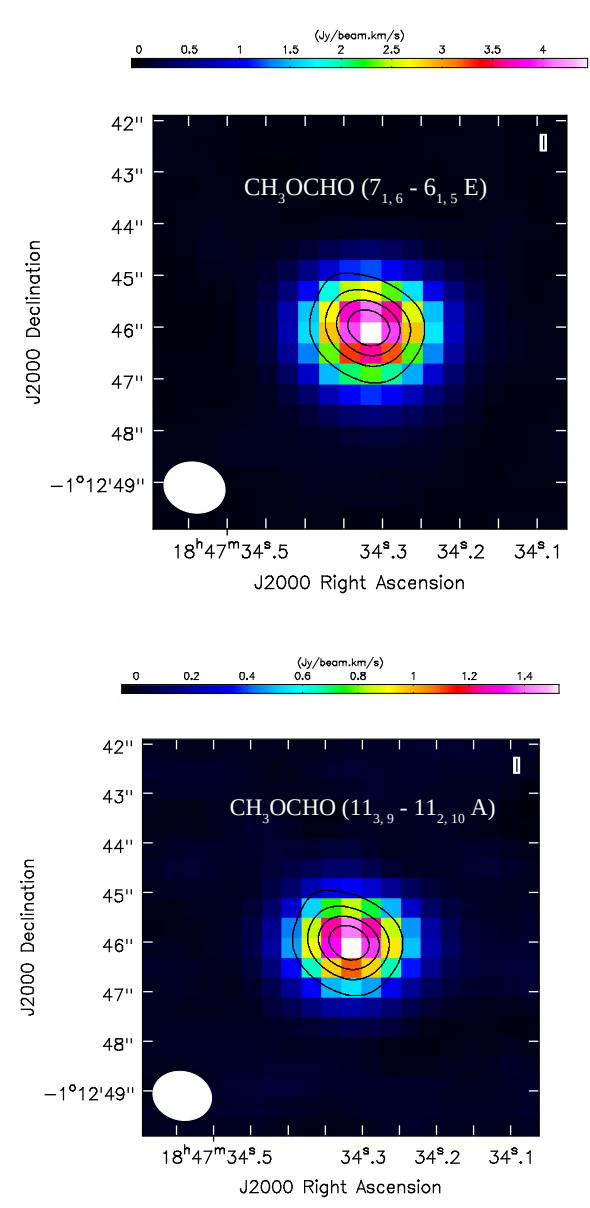
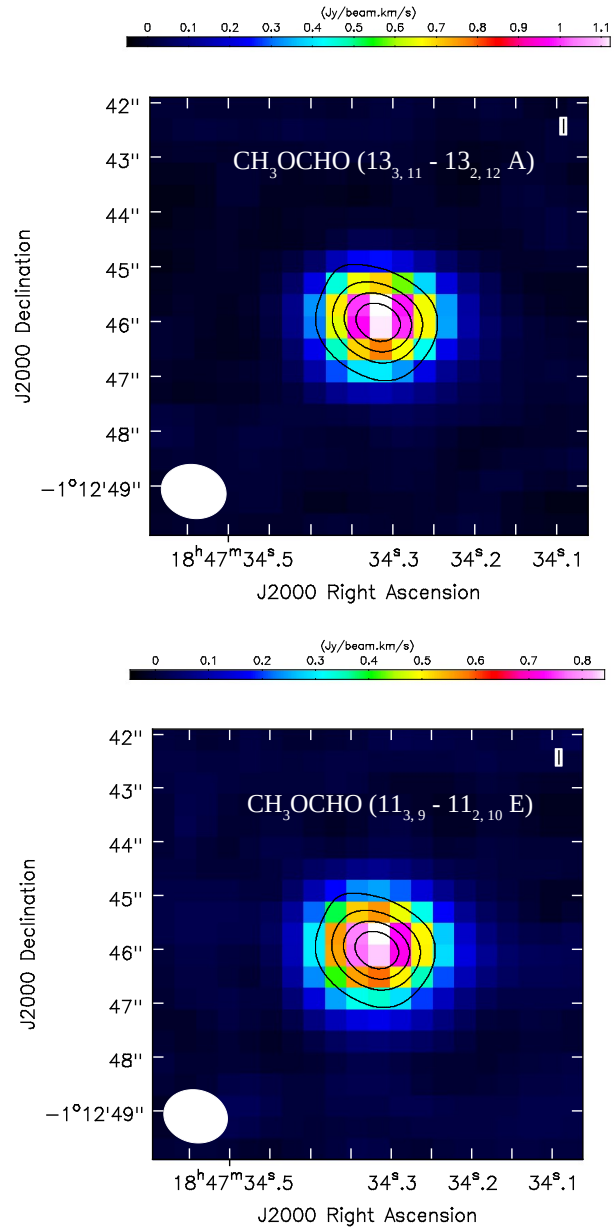

Figure A10. Moment 0 of the integrated intensity distribution (color) of $\mathrm{CH}_{3} \mathrm{OCHO}$ transitions is overlaid on the $3.1 \mathrm{~mm}$ continuum emission (black contours). Contour levels are at $20 \%, 40 \%, 60 \%$, and $80 \%$ of the peak flux of the continuum image. The synthesized beam is shown in the lower left-hand corner of each figure. 

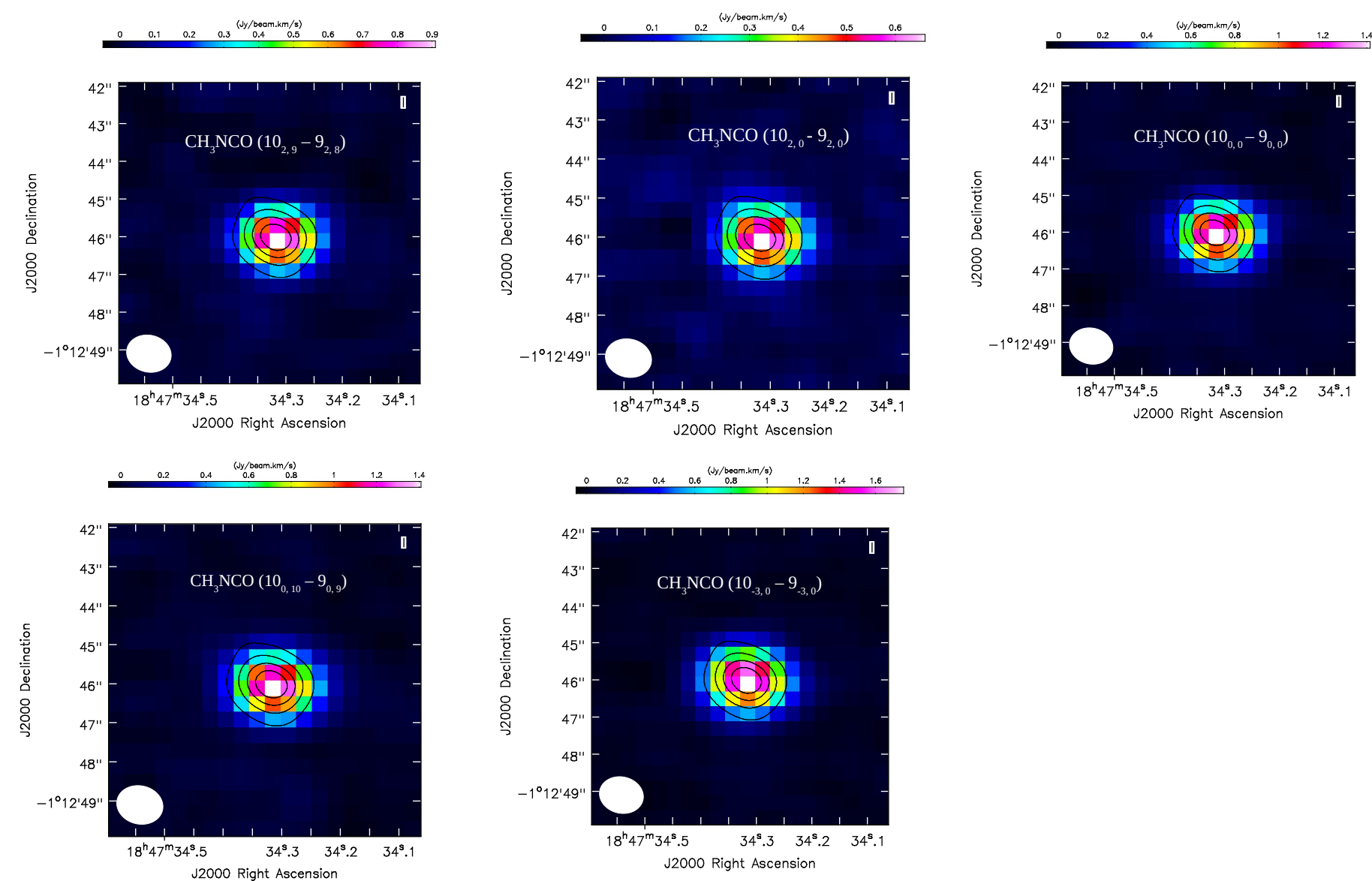

Figure A11. Moment 0 of the integrated intensity distribution (color) of $\mathrm{CH}_{3} \mathrm{NCO}$ transitions is overlaid on the 3.1 mm continuum emission (black contours). Contour levels are at 20\%,40\%, $60 \%$, and $80 \%$ of the peak flux of the continuum image. The synthesized beam is shown in the lower left-hand corner of each figure.
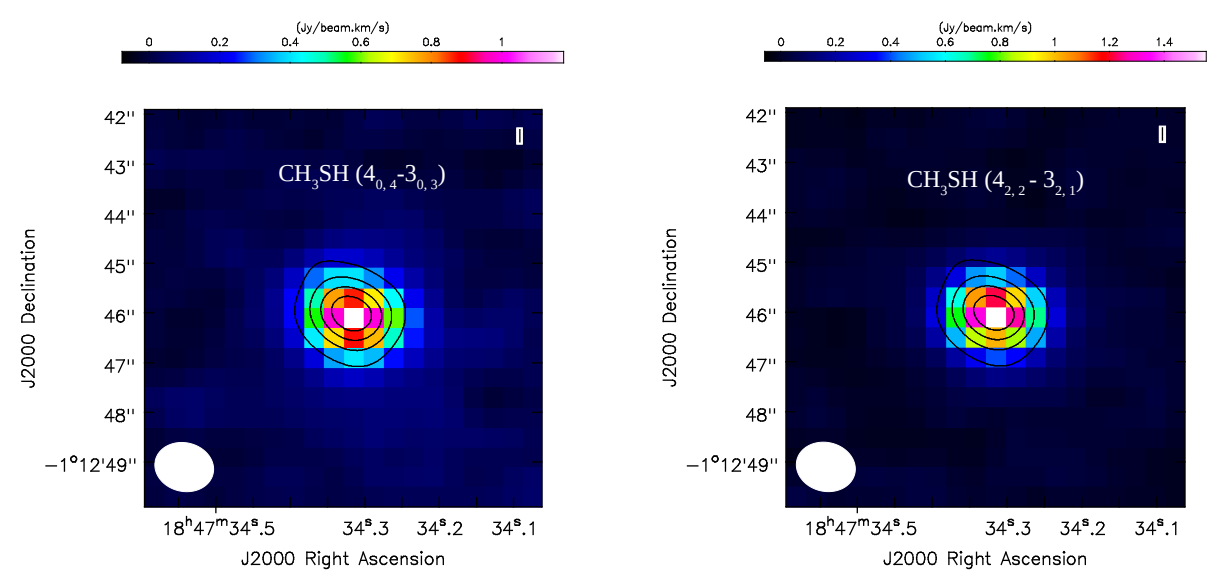

Figure A12. Moment 0 of the integrated intensity distribution (color) of $\mathrm{CH}_{3} \mathrm{SH}$ transitions is overlaid on the 3.1 mm continuum emission (black contours). Contour levels are at $20 \%, 40 \%, 60 \%$, and $80 \%$ of the peak flux of the continuum image. The synthesized beam is shown in the lower left-hand corner of each figure. Multiple transitions (101.13915/101.13965 GHz) appear in $\mathrm{CH}_{3} \mathrm{SH} 4_{0,4}-3_{0,3}$ moment maps. Similarly multiple transitions $(101.16715 / 101.16830 \mathrm{GHz})$ appear in $\mathrm{CH}_{3} \mathrm{SH}_{2,2}-3_{2,1}$ moment maps. 
Table B1. Emitting region of observed transitions of COMs

\begin{tabular}{|c|c|c|c|}
\hline Species & $\begin{array}{c}\text { Transition } \\
\text { Frequency } \\
\quad(\mathrm{GHz})\end{array}$ & $\begin{array}{l}\text { Emitting } \\
\text { region } \\
\left({ }^{\prime \prime}\right)\end{array}$ & $\begin{array}{c}\text { Upper State } \\
\text { Energy } \\
(\mathrm{K})\end{array}$ \\
\hline \multirow[t]{3}{*}{$\mathrm{CH}_{3} \mathrm{SH}$} & $101.13915^{a}$ & $0.89 \pm 0.06$ & 12.14 \\
\hline & $101.15999^{b}$ & $0.80 \pm 0.02$ & 52.39 \\
\hline & $101.16830^{c}$ & $0.80 \pm 0.05$ & 30.27 \\
\hline \multirow[t]{7}{*}{$\mathrm{CH}_{3} \mathrm{OH}$} & 86.61557 & $1.35 \pm 0.01$ & 102.70 \\
\hline & 86.90291 & $1.35 \pm 0.02$ & 102.72 \\
\hline & 88.59478 & $1.29 \pm 0.02$ & 328.28 \\
\hline & 88.93997 & $1.27 \pm 0.01$ & 328.28 \\
\hline & 101.12685 & $1.14 \pm 0.03$ & 60.73 \\
\hline & 101.29341 & $1.21 \pm 0.02$ & 90.91 \\
\hline & 101.46980 & $1.23 \pm 0.03$ & 109.49 \\
\hline \multirow[t]{5}{*}{$\mathrm{CH}_{3} \mathrm{NCO}$} & 86.68019 & $0.82 \pm 0.01$ & 22.82 \\
\hline & 86.68655 & $0.83 \pm 0.02$ & 34.97 \\
\hline & 86.78077 & $0.78 \pm 0.02$ & 46.73 \\
\hline & 86.80502 & $0.77 \pm 0.01$ & 46.74 \\
\hline & 87.01607 & $0.76 \pm 0.02$ & 58.88 \\
\hline \multirow[t]{7}{*}{$\mathrm{CH}_{3} \mathrm{OCHO}$} & 88.6869 & $1.37 \pm 0.04$ & 44.97 \\
\hline & 88.72326 & $1.10 \pm 0.03$ & 44.96 \\
\hline & 88.84318 & $1.36 \pm 0.02$ & 17.96 \\
\hline & 88.85160 & $1.42 \pm 0.02$ & 17.94 \\
\hline & 88.86241 & $1.15 \pm 0.04$ & 207.10 \\
\hline & 101.37054 & $1.46 \pm 0.05$ & 59.64 \\
\hline & 101.41474 & $1.33 \pm 0.06$ & 59.63 \\
\hline $\mathrm{SO}_{2}$ & 86.63908 & $0.71 \pm 0.01$ & 55.20 \\
\hline $\mathrm{H}_{2} \mathrm{CO}$ & 101.33299 & $1.31 \pm 0.02$ & 87.57 \\
\hline
\end{tabular}

NOTES: ${ }^{a}$ multiple transitions: $101.1391 \overline{\overline{5} / 101.13965 \mathrm{GHz},{ }^{b} \text { multiple transitions: } 101.15999 / 101.15933 / 101.16066 / 101.16069}$ $\mathrm{GHz},{ }^{c}$ multiple transitions: $101.16715 / 101.16830 \mathrm{GHz}$.

B. SUMMARY OF THE EMITTING REGIONS OF THE OBSERVED SPECIES. 